\title{
CLASH-VLT: Strangulation of cluster galaxies in MACS J0416.1-2403 as seen from their chemical enrichment ${ }^{\star}$
}

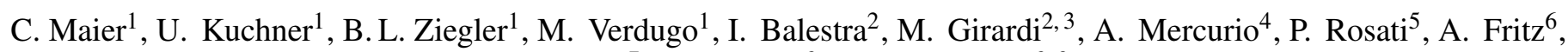 \\ C. Grillo ${ }^{7}$, M. Nonino ${ }^{2}$, and B. Sartoris ${ }^{2,3}$
}

${ }^{1}$ University of Vienna, Department of Astrophysics, Tuerkenschanzstrasse 17, 1180 Vienna, Austria e-mail: christian.maier@univie.ac.at

2 INAF-Osservatorio Astronomico di Trieste, via G. B. Tiepolo 11, 34133 Trieste, Italy

3 Dipartimento di Fisica, Università degli Studi di Trieste, via Tiepolo 11, 34143 Trieste, Italy

4 INAF-Osservatorio Astronomico di Capodimonte, via Moiariello 16, 80131 Napoli, Italy

5 Dipartimento di Fisica e Scienze della Terra, Università di Ferrara, via Saragat 1, 44122 Ferrara, Italy

6 INAF-Istituto di Astrofisica Spaziale e Fisica cosmica (IASF) Milano, via Bassini 15, 20133 Milano, Italy

7 Dark Cosmology Centre, Niels Bohr Institute, University of Copenhagen, Juliane Maries Vej 30, 2100 Copenhagen, Denmark

Received 29 January 2016 / Accepted 18 March 2016

\begin{abstract}
Aims. Environmental effects gain importance as large scale structures in the Universe develop with time and have become the dominant mechanism for quenching galaxies of intermediate and low stellar masses at lower redshifts. Therefore, clusters of galaxies at $z<0.5$ are the sites where environmental effects are expected to be more pronounced and more easily observed with present-day large telescopes.

Methods. We explore the Frontier Fields cluster MACS J0416.1-2403 at $z=0.3972$ with VIMOS/VLT spectroscopy from the CLASH-VLT survey covering a region that corresponds to almost three virial radii. We measure fluxes of $\mathrm{H} \beta$, [O III] $\lambda 5007, \mathrm{H} \alpha$, and [N II] $\lambda 6584$ emission lines of cluster members enabling us to unambiguously derive $\mathrm{O} / \mathrm{H}$ gas metallicities, and also star formation rates from extinction-corrected $\mathrm{H} \alpha$ fluxes. We compare our cluster galaxy sample with a field sample at $z \sim 0.4$ drawn from zCOSMOS.

Results. The 76 galaxies of our cluster sample follow the star-forming metallicity sequence in a diagnostic diagram disentangling ionizing sources. For intermediate masses we find a similar distribution of cluster and field galaxies in the mass vs. metallicity and mass vs. sSFR diagrams. An in-depth investigation furthermore reveals that bulge-dominated cluster galaxies have on average lower sSFRs and higher $\mathrm{O} / \mathrm{Hs}$ than their disk-dominated counterparts. We use the location of galaxies in the projected velocity vs. position phase-space to separate our cluster sample into a region of objects accreted longer ago and a region of recently accreted and infalling galaxies. We find a higher fraction of accreted metal-rich galaxies (63\%) compared to the fraction of $28 \%$ of metal-rich galaxies in the infalling regions. Intermediate-mass galaxies $\left(9.2<\log \left(M / M_{\odot}\right)<10.2\right)$ falling into the cluster for the first time are found to be in agreement with predictions of the fundamental metallicity relation. In contrast, for already accreted star-forming galaxies of similar masses, we find on average metallicities higher than predicted by the models. This trend is intensified for accreted cluster galaxies of the lowest mass bin $\left(\log \left(M / M_{\odot}\right)<9.2\right)$, that display metallicities two to three times higher than predicted by models with primordial gas inflow. Environmental effects therefore strongly influence gas regulations and control gas metallicities of $\log \left(M / M_{\odot}\right)<10.2$ cluster galaxies. We also investigate chemical evolutionary paths of model galaxies with and without inflow of gas showing that strangulation is needed to explain the higher metallicities of accreted cluster galaxies. Our results favor a strangulation scenario in which gas inflow stops for galaxies with $\log \left(M / M_{\odot}\right)<10.2$ when accreted by the cluster.
\end{abstract}

Key words. galaxies: evolution - galaxies: clusters: individual: MACS J0416.1-2403 - galaxies: star formation galaxies: abundances - galaxies: structure

\section{Introduction}

Gas-phase metallicites in galaxies are regulated by the complex interplay between star formation, accretion of metal-poor gas and galactic outflows of processed gas in galaxies, representing their current state of chemical enrichment. Comprehensive studies of large samples in the local Universe have established a tight relation between stellar mass and metallicity showing that the brighter and hence more massive a galaxy is, the greater is its gas metallicity as measured by the $\mathrm{O} / \mathrm{H}$ abundance (e.g., using

\footnotetext{
* Based on observations obtained at the European Southern Observatory (ESO) Very Large Telescope (VLT), Paranal, Chile; ESO large program 186.A-0798.
}

SDSS, Tremonti et al. 2004; Kewley \& Ellison 2008). One explanation for the origin of this relation has been summarized by Lilly et al. (2013, Li13 in the following), and is known as the bathtub model. The star formation rate (SFR) is thereby closely linked to the mass of gas in the galaxy modulated by infalling gas and outflows driven by supernovae winds. In this scenario a relation between metallicity, SFR, and stellar mass can be derived. Several authors have studied the role of the SFR and of the specific SFR $\left(s S F R=S F R / M_{*}\right)$ in this context and have presented links between SFR and metallicity (e.g., Ellison et al. 2008; Lara-López et al. 2010), finally claiming an epoch-independent fundamental metallicity relation (FMR) between metallicity, mass, and $\mathrm{SFR}, Z\left(M_{*}, S F R\right)$, expected to 
be applicable at all redshifts (Mannucci et al. 2010). Recenty, Salim et al. (2014) reconciled conflicting results regarding this SFR dependence showing that the metallicity is anti-correlated with SSFR for SDSS local galaxies, regardless of the metallicity and SFR indicators used.

The evolution of galaxies is governed internally by their stellar mass and externally influenced by their environment (e.g., Peng et al. 2010a, 2012) made visible through well-established changes in the observable properties of cluster galaxies that affect the star formation histories (SFHs), gas and stellar components. Therefore, examining the interrelationships between stellar mass, environment, metallicity, and stellar structure, and their evolution with time will reveal the physical processes that control the evolution of galaxies. Since environmental effects get stronger at $z<0.5$ as large-scale structure in the Universe develops (see, e.g., Fig. 15 in Peng et al. 2010a), clusters of galaxies at $z<0.5$ are the sites where influences of the environment should be detected more easily with the available present-day large telescopes. It is well established that specific cluster phenomena influence star formation and the exchange of gas, for example, by supressing gas inflow. Star formation and gas inflow influence gas metallicities; therefore, it is expected that gas metallicities depend on environment.

According to previous works in the local Universe, using SDSS galaxies, little effect of the environment on the massmetallicity relation (MZR) was found. Defining environment by local densities, Mouhcine et al. (2007) reported variation in metallicity, at a given mass, ranging from 0.02 dex for massive to $0.08 \mathrm{dex}$ for galaxies with $\log M / M_{\odot}<9.5$ occurring over a contrast factor of 100 . In their environmental studies of central and satellite SDSS galaxies, Pasquali et al. (2012) and Peng \& Maiolino (2014) both reported an average metallicity of satellites higher than that of centrals, especially for low stellar mass galaxies; this difference in metallicity was found to gradually disappear towards higher stellar masses. Another investigation of 1318 galaxies in cluster environments found an increase of 0.04 dex in oxygen abundances for galaxies in clusters compared to the field (Ellison et al. 2009). This was confirmed numerically by Davé et al. (2011), who used cosmological hydrodynamic simulations to investigate environmental effects on the MZR, reporting a small enhancement in the mean MZR of galaxies in high-density regions of $\sim 0.05 \mathrm{dex}$. On the other hand, Cooper et al. (2008) used the SDSS sample and claimed that there is a stronger relationship between metallicity and environment (largely driven by galaxies in high-density regions such as groups and clusters), such that more metal-rich galaxies favor regions of higher overdensity.

The current state and gas content of galaxies is furthermore expected to be related to their morphologies. The importance of structural parameters such as compactness and surface mass densities in setting the gas properties has been recognized before (Saintonge et al. 2012). Galaxies with higher surface mass densities have transformed more of their gas into stars over the course of their SFH, thereby enhancing the overall gas-phase metallicity (Kauffmann et al. 2003b). The dependence of the MZR on structural parameters leaves its imprint on the scatter of the relation. Tremonti et al. (2004) related higher stellar mass surface densities to higher metallicities at fixed stellar mass. Additionally, Ellison et al. (2008) found that at fixed stellar masses, galaxies with larger sizes (i.e., with lower stellar mass surface densities) have lower metallicities. On the theroretical side, Calura et al. (2009) studied the MZR by means of chemical evolution models of different morphological types. Their results indicate that the observed MZR is reproduced by considering different morphological mixes at different redshifts. The varying strength of a central bulge governs the classification scheme introduced by Hubble (1926). While connections between the presence of a bulge and quenching of star formation have been reported (e.g., Saintonge et al. 2012, suggesting that the main driver for gas depletion in galaxies is bulge mass), the physical mechanisms responsible for this star formation shutoff and the enhancement of measured gas metallicities is not yet fully understood. Connecting the gas metallicities with mass, SFRs, and morphologies as a function of environment and redshift may therefore lead to increased knowledge of the temporal evolution of the chemical properties of the stellar populations and allow a more qualitative look into the nature of the galaxies building the MZR.

We can now extend these investigations to intermediate redshifts, measuring $\mathrm{O} / \mathrm{H}$ abundances in a very massive cluster at $z \sim 0.4$, and contrasting them to comparable measurements of zCOSMOS field galaxies at similar redshifts. We do this by using the two connected relations, the MZR and the mass(s)SFR relation, presenting evolutionary effects between $z \sim 0.4$ and the local Universe. To estimate the chemical abundances, a number of diagnostics have been developed based on strong emission lines (ELs), [O II] $\lambda 3727, \mathrm{H} \beta,[\mathrm{O} \mathrm{III]} \lambda \lambda 5007, \mathrm{H} \alpha$, and $[\mathrm{NII}] \lambda 6584$. At higher redshifts, these ELs move to the nearinfrared, with $\mathrm{H} \alpha$ and [NII] $\lambda 6584$ already shifting beyond the optical above redshifts of $\sim 0.5$, requiring near-infrared spectroscopy to observe these ELs (e.g., Maier et al. 2004, 2005, $2006,2014,2015)$. Thus, the epoch we probe is the highest redshift at which all these ELs can be observed with the same optical spectrograph without the complication of obtaining a good relative calibration between spectra from optical and near-infrared spectrographs.

The paper is structured as follows. In Sect. 2 we present the selection of the cluster EL galaxies at $z \sim 0.4$ and their VIMOS spectroscopy. We also discuss the comparison field samples of zCOSMOS and SDSS galaxies. In Sect. 3 we investigate the active galactic nucleus (AGN) contribution and present the derivation of SFRs, metallicities, morphological parameters, and stellar masses of the 76 cluster galaxies we observed. In Sect. 4 we present the MZR and mass-sSFR relation at $z \sim 0.4$, and investigate their dependence on environment and morphological parameters. In Sect. 5 we discuss how the cluster environment affects the chemical enrichment. We investigate how the SFR impacts the MZR in the cluster environment, and how this compares with predictions of the FMR. Several pieces of evidence for a strangulation scenario for intermediate- and lowmass cluster galaxies are discussed. Finally in Sect. 6 we summarize our conclusions. A concordance-cosmology with $H_{0}=$ $70 \mathrm{~km} \mathrm{~s}^{-1} \mathrm{Mpc}^{-1}, \Omega_{0}=0.25, \Omega_{\Lambda}=0.75$ is used throughout this paper. We assume a Salpeter (Salpeter 1955) initial mass function (IMF) for all derived stellar masses and SFRs and correct existing measurements used in this paper to a Salpeter IMF. We note that metallicity and abundance will be taken to denote oxygen abundance, $\mathrm{O} / \mathrm{H}$, throughout this paper, unless otherwise specified. In addition, we use dex throughout to denote the antilogarithm, i.e., 0.3 dex is a factor of two.

\section{The data: cluster and field galaxies at $z \sim 0.4$}

\subsection{The MAC0416 cluster}

MACS J0416.1-2403 (hereafter MAC0416) is a massive, X-ray-luminous (Ebeling et al. 2001) galaxy cluster at $z=$ 0.3972 . MAC0416 is one of 25 clusters in the HST multicycle 

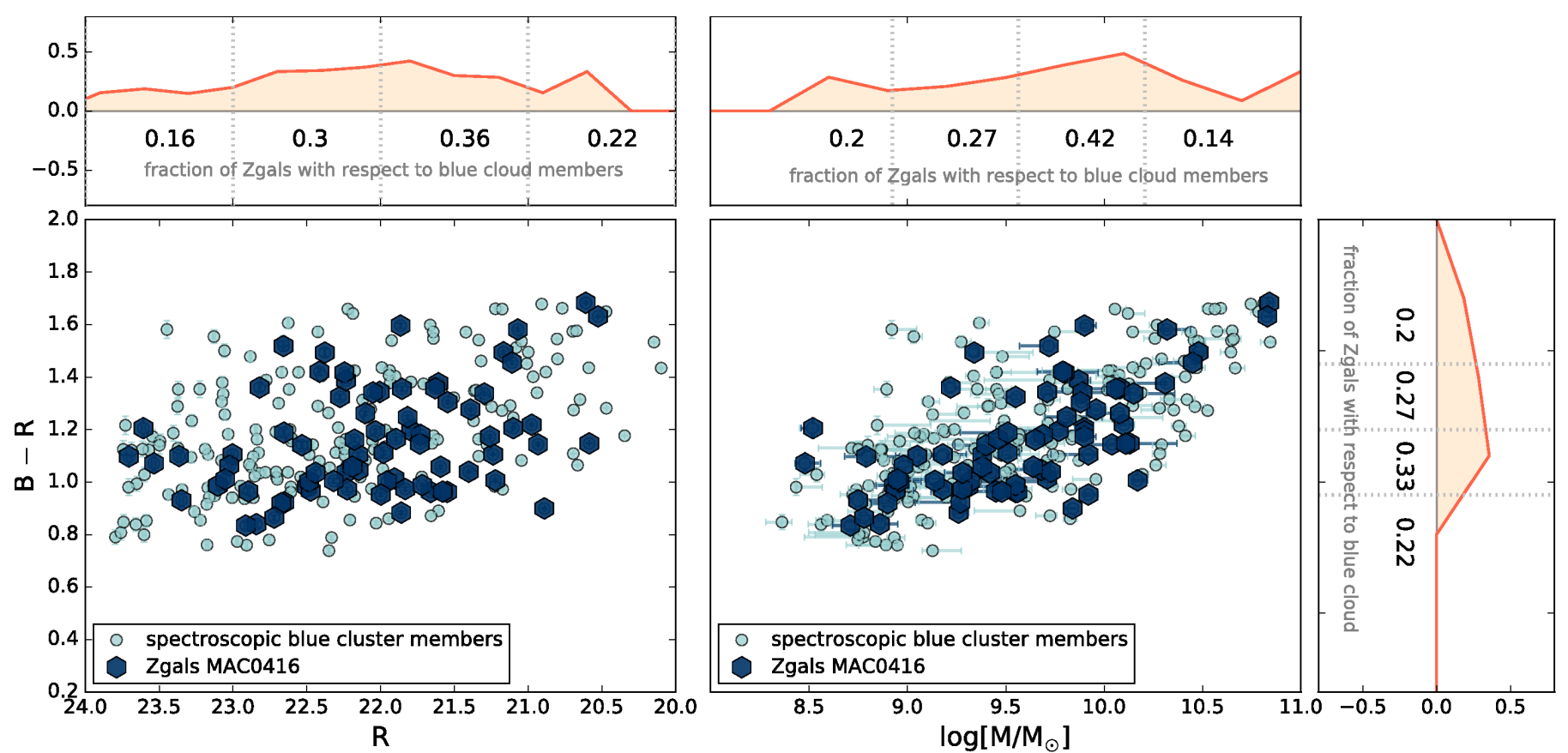

Fig. 1. Color-luminosity (left) and color-mass (right) diagrams for MAC0416 cluster galaxies at $z \sim 0.4$ with spectroscopic redshifts. Cyan data points are the spectroscopic confirmed MAC0416 blue cluster members, representative of the star-forming blue cloud galaxies, while the 76 galaxies used for this metallicity study (Zgals sample) are shown in blue. The red lines and numbers indicate the fraction of Zgals objects (blue symbols) with respect to the parent sample (cyan points). We achieve a uniform coverage at intermediate luminosities/masses. The lowest and highest luminosity/mass bins are under-represented by our sample.

treasury program "Cluster Lensing And Supernova survey with Hubble" (CLASH, Postman et al. 2012). The mass profile measured from the galaxy dynamics (Balestra et al. 2016, hereafter B16) reveals a complex dynamical state of this cluster that can be explained by a pre-collisional phase scenario. The inner regions of the cluster are nevertheless represented best by a simple isothermal sphere (SIS) model, as described in B16.

CLASH-VLT (Rosati et al. 2014), the spectroscopic followup of 13 CLASH clusters at $0.3<z<0.6$, provides spectra over an area of $25 \times 25$ square arcmin, using the low resolution blue (hereafter LB) and medium resolution (MR) grisms of VIMOS. The details of the LB and MR VIMOS observations and redshift determinations of MAC0416 are given in B16. The observations and data reduction of the MR pointings used for our SFR and metallicity measurements are described in Sect. 2.1.2. High quality Subaru Suprime-Cam BRz imaging covers the VIMOS field that is $25 \times 25$ square arcminutes in area (Umetsu et al. 2014). For our metallicity study we selected this particular cluster from the CLASH sample owing to its ideal redshift $(z \sim 0.4)$, which places all five strong ELs in a region not affected by night skylines, allowing measurements of all ELs needed in one spectrum using the MR grism of VIMOS.

The identification of cluster members was described in B16, adopting as the center of the cluster the position of one of the two brightest cluster galaxies (BCGs), namely the NE-BCG position, which coincides with the peak of the X-ray emission (see Ogrean et al. 2015). Balestra et al. (2016) obtained a velocity dispersion $\sigma \sim 1000 \mathrm{~km} \mathrm{~s}^{-1}$, virial radius $R_{200} \sim 1.8 \mathrm{Mpc}$, and a virial mass of $M_{200} \sim 1 \times 10^{15} M_{\odot}$ for MAC0416, where the "200" subscript refers to the radius where the average density is 200 times the mean density of the Universe at the cluster redshift. We consider as cluster members all galaxies within a larger range of $\pm 3 \sigma$ of the velocity dispersion because we want to study environmental effects by dividing the sample into "infalling" and "accreted longer ago" cluster galaxies, as described in Sect. 5.

\subsubsection{Selection of the cluster galaxies for our metallicity study}

The target selection for the LB VIMOS pointings was based on color-color diagrams (as described in B16). The spectroscopic redshifts derived from the [O II] $\lambda 3727$ ELs as delivered by the VIMOS LB grism observations allowed us to select an appropriate target sample, with ELs free of strong telluric emission, for the MR grism observations that provide higher resolution and larger (including redder) wavelength coverage than the LB spectra. The target sample contained 165 blue cluster member galaxies with the four ELs $\mathrm{H} \beta$, [O III] $\lambda 5007, \mathrm{H} \alpha$, and [N II] $\lambda 6584$, which are necessary for the derivation of reliable metallicities, free of contamination by night skylines. Eighty-two galaxies in this sample were observed using the MR grism, 60 of which have a signal-to-noise ratio $(\mathrm{S} / \mathrm{N})$ higher than two in the weakest EL detected (H $\beta$, [O III] $\lambda 5007$, or [N II] $\lambda 6584)$. Additionally, some galaxies without LB spectra were selected based on color-color criteria and were observed with the MR grism without being part of the parent sample of 165 blue cluster members. Sixteen of these galaxies turned out to have five ELs with high enough $\mathrm{S} / \mathrm{N}$ for metallicity studies resulting in a total sample of 76 cluster galaxies with five ELs detected (sample Zgals).

Figure 1 demonstrates the uniform coverage of our sample Zgals with respect to the parent sample of all spectroscopic blue cloud galaxies in both luminosities and masses. The figure shows the color-magnitude and color-mass diagrams of the spectroscopic member galaxies of the blue cloud (cyan filled circles) and the 76 Zgals objects used for our metallicity study (blue symbols). The fractions indicate that our Zgals sample is a good representation of the overall blue (star-forming) population of 
Table 1. Demonstration of the coverage of our sample.

\begin{tabular}{|c|c|c|c|c|c|c|c|c|c|c|c|c|}
\hline \multirow[b]{2}{*}{ Bins } & \multicolumn{4}{|c|}{ Luminosity ( $R$ magnitude) } & \multicolumn{4}{|c|}{ Stellar mass } & \multicolumn{4}{|c|}{ Color $B-R$} \\
\hline & faint & $\rightarrow$ & $\rightarrow$ & bright & low & $\rightarrow$ & $\rightarrow$ & high & blue & $\rightarrow$ & $\rightarrow$ & red \\
\hline Members & $21 \%$ & $37 \%$ & $32 \%$ & $10 \%$ & $15 \%$ & $41 \%$ & $29 \%$ & $16 \%$ & $13 \%$ & $47 \%$ & $25 \%$ & $15 \%$ \\
\hline Zgals & $12 \%$ & $39 \%$ & $41 \%$ & $8 \%$ & $11 \%$ & $39 \%$ & $42 \%$ & $8 \%$ & $11 \%$ & $55 \%$ & $24 \%$ & $11 \%$ \\
\hline Fraction & $16 \%$ & $30 \%$ & $36 \%$ & $22 \%$ & $20 \%$ & $27 \%$ & $42 \%$ & $14 \%$ & $22 \%$ & $33 \%$ & $27 \%$ & $20 \%$ \\
\hline
\end{tabular}

Notes. First row: percentage of spectroscopic members of MAC0416 representative of the blue cloud in the given bins in luminosity, mass, and color. Second row: selected Zgals objects for this metallicity study in the given bins in luminosity, mass, and color. Third row: the fractions of Zgals objects in different luminosity/mass/color bins with respect to the parent sample of blue cluster members (numbers shown in Fig. 1).

cluster galaxies, especially at intermediate masses. Table 1 lists the percentages of all spectroscopic members representative for the blue cloud (first row) in four luminosity, mass, and color bins, the percentage of the Zgals objects in the same bins (second row), and their fractions (third row) as visualized in Fig. 1.

\subsubsection{MR VIMOS observations and data reduction}

The main target galaxies were observed in four VIMOS pointings with the MR grism and GG475 filter with exposure times of 60 min each, and masks designed with 1 arcsec slits. The covered wavelength range was 480-1000 $\mathrm{nm}$ with a resolution $R=580$, thereby including ELs from [O II] $\lambda 3727$ to [N II] $\lambda 6584$ of $z \sim 0.4$ galaxies.

Data reduction of the VIMOS spectra of MAC0416 galaxies was carried out using the Vimos Interactive Pipeline Graphical Interface (VIPGI; Scodeggio et al. 2005) software package. VIPGI uses automated procedures for bias subtraction, flux and wavelength calibration of the spectra, identification of objects in the slit profile, and extraction of the one-dimensional spectra. Wavelength calibration uses a HeNeAr arc lamp exposure obtained immediately following the science exposures. The standard star LTT-1788 was used for flux calibration. The determination of redshifts of target galaxies is unambiguous owing to the five ELs observed with the MR grism of VIMOS.

\subsection{Comparison sample: zCOSMOS field galaxies at $z \sim 0.4$}

As our comparison sample of field galaxies, we selected 93 zCOSMOS-bright (Lilly et al. 2007, 2009) objects at similar redshifts to the cluster galaxies, namely $0.373<z<0.413$, ensuring that all ELs are observed free of strong OH night skylines. These objects have four ELs measured with VIMOS $-\mathrm{H} \beta$, [O III] $\lambda$ 5007, $\mathrm{H} \alpha$, and [N II] $\lambda 6584-$ and stellar masses derived from the SED fitting based on 30 COSMOS bands (Ilbert et al. 2009). For details of the measurements of zCOSMOS EL fluxes we refer to Maier et al. (2009), Pérez-Montero et al. (2013), and Maier et al. (2015).

\subsection{Local comparison sample: SDSS}

We selected a local comparison sample of SDSS galaxies from the DR4 release, Garching repository ${ }^{1}$, in the redshift range $0.04<z<0.08$. The lower redshift limit of $z=0.04$ was chosen following Kewley et al. (2005) to reduce the systematic and random errors in the SFRs that arise from aperture effects due to the 3 arcsec size of the SDSS fibers. Duplicate objects and SDSS galaxies on problematic or special plates were excluded from the

\footnotetext{
1 http://www . mpa-garching.mpg.de/SDSS/DR4/
}

SDSS sample; since this exclusion was based solely on position on the sky, it should not be related to any galaxy properties.

We excluded AGNs from the SDSS sample using the [O III] $\lambda 5007 / \mathrm{H} \beta$ vs. [N II] $\lambda 6584 / \mathrm{H} \alpha$ diagnostic diagram, excluding objects that satisfy Eq. (1) of Kauffmann et al. (2003a). The evolution of the sSFR shows an average decrease of a factor of about two from $z \sim 0.4$ until the epoch of the SDSS sample, at redshifts $0.04<z<0.08$ (see also Sect. 4.1). Therefore, a lower limit in SFR of $0.3 M_{\odot} /$ yr for our Zgals MAC0416 sample at $z \sim 0.4$ corresponds to a lower limit of $0.15 M_{\odot} / \mathrm{yr}$ for the SDSS sample. To ensure a consistent comparison of the physical properties of the SDSS and Zgals samples, we applied an additional $S / N>10$ cut in $\mathrm{H} \alpha$ for SDSS galaxies as was applied for the Zgals sample, and only SDSS galaxies with SFRs larger than $0.15 M_{\odot} / \mathrm{yr}$ were used. Therefore, it should be noted that this sample is slightly different from other local comparison SDSS samples often used in the literature such as the samples of Tremonti et al. (2004) or Mannucci et al. (2010).

\section{Measurements}

\subsection{EL fluxes}

The EL fluxes of MAC0416 cluster member galaxies were measured interactively using the package splot in IRAF. The flux errors were usually dominated by systematic uncertainties in establishing the local continuum, which was conservatively estimated by exploring rather extreme possibilities. Following the recommendation of Kobulnicky et al. (1999) for the statistical correction of $\mathrm{H} \beta$ EL flux measurements from global galaxy spectra, we assumed an average underlying stellar absorption in $\mathrm{H} \beta$ of $3 \pm 2 \AA$ and corrected the equivalent width (EW) of this line by this amount, thereby increasing the $\mathrm{H} \beta$ line flux. This correction is often used in the literature for data with similar spectral resolution to that used in our sample (e.g., Lilly et al. 2003; Maier et al. 2005), and the range of $1 \AA$ to $5 \AA$ for the $\mathrm{EW}$ in $\mathrm{H} \beta$ absorption corresponds to the values found for the entire range of typical ages of the stellar population (see, e.g., Kobulnicky et al. 1999; Maier et al. 2015). The uncertainty of $2 \AA$ acts as an additional error term for lower $\mathrm{S} / \mathrm{N}$ in $\mathrm{H} \beta$. We use this uncertainty and account for it in the total error budget when computing the error bars for the corrected $\mathrm{H} \beta$ EL fluxes and derived quantities (see Table A.1). We note that all $\mathrm{H} \alpha$ ELs of Zgals cluster galaxies have a $S / N>10$, and 70 out of 76 galaxies (92\% of the sample) have a $S / N>3$ in [N II] $\lambda 6584$.

\subsection{Star formation or AGNs?}

We use the BPT (Baldwin, Phillips \&Terlevich; Baldwin et al. 1981) diagram shown in Fig. 2, to establish whether the source 


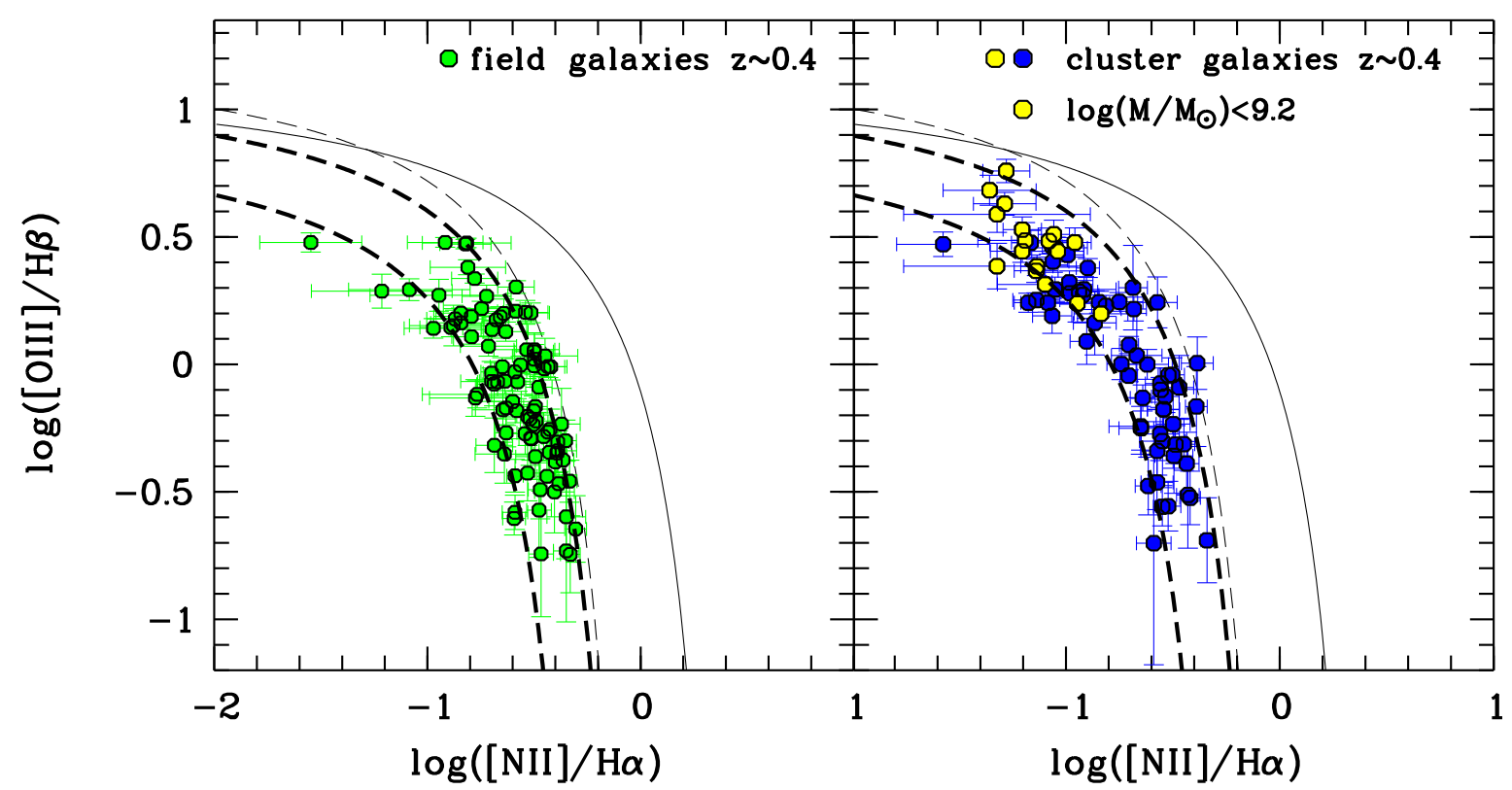

Fig. 2. BPT (Baldwin et al. 1981) diagnostic diagram used to distinguish star formation-dominated galaxies from AGNs. The left diagram shows the field galaxies at $z \sim 0.4$, and the right panel the Zgals cluster members of MAC0416 (cluster galaxies of similar high masses to the field comparison sample are depicted in blue). Both the field and cluster galaxies at $z \sim 0.4$ lie under and to the left of the theoretical curve (solid) of Kewley et al. (2001) and of the empirical curve (thin dashed) of Kauffmann et al. (2003a), which separate star-forming galaxies (below and left of the curves) from AGNs (above and right of the curves), indicating that in all of them the dominant source of ionization in the gas is recent star formation. Most galaxies lie on the star-forming metallicity sequence with rough boundaries indicated by the thick dashed lines.

of gas ionization is of stellar origin or is instead associated with AGN activity. The MAC0416 Zgals cluster galaxies and also all zCOSMOS field galaxies at $z \sim 0.4$ lie under and to the left of the theoretical curve (solid) of Kewley et al. (2001) and the empirical curve (dashed) of Kauffmann et al. (2003a), which separate star-forming galaxies from AGNs. This indicates that in all of them the dominant source of ionization of the gas is recent star formation. Moreover, most galaxies lie on the starforming abundance sequence with rough boundaries indicated by the thick dashed lines. zCOSMOS field galaxies, which all have $\log \left(M / M_{\odot}\right)>9.2$, and Zgals cluster galaxies of similar masses (blue symbols) lie in a similar, lower part of the BPT diagram. Cluster galaxies with lower masses $\log \left(M / M_{\odot}\right)<9.2$ (yellow symbols) have typically higher [O III $] \lambda 5007 / \mathrm{H} \beta$ ratios, which correspond to lower metallicities (cf. Fig. A.1 in Maier et al. 2015).

\subsection{Oxygen abundances}

Equation (4) in Kewley et al. (2013), $12+\log (\mathrm{O} / \mathrm{H})=8.97-$ $0.32 \times \log (([\mathrm{OIII}] / \mathrm{H} \beta) /([\mathrm{NII}] / \mathrm{H} \alpha))$, corresponds to the Pettini \& Pagel (2004) O3N2 calibration for the Kewley \& Dopita (2002) metallicity scale. The only difference from the Pettini \& Pagel (2004) O3N2 calibration is that the first (normalization) factor is 8.97 instead of 8.73 . We use this equation to consistently compute metallicities for the Zgals MAC0416 cluster objects, the zCOSMOS field galaxies at $z \sim 0.4$, and for the SDSS sample.

There are several reasons for this choice of metallicity calibration. First, the zCOSMOS sample at $z \sim 0.4$ does not have [OII] measured, but only the other four ELs at redder wavelengths. Second, it is more straightforward to compute O3N2 metallicities than to use a $\chi^{2}$ method, which is based on the comparison of five measured ELs with theoretical predictions of the fluxes for given metallicities and extinction values. The latter method can be affected by gradients and degeneracies of the theoretical models (see discussion in Maier et al. 2005). Third, using $\mathrm{O} 3 \mathrm{~N} 2$ the comparison with other literature data is easier, because the $\chi^{2}$ approach of simultaneously fitting all five strong ELs and using theoretical models to generate a probability distribution of metallicities and statistically estimate the oxygen abundances is very seldom used in the literature. Fourth, as discussed above in Sect. 3.2, most galaxies in this study follow a star-forming abundance sequence in the BPT diagram, indicating that some line ratios are related to each other, which the $\chi^{2}$ method does not take into account.

\subsection{Stellar masses}

Stellar masses of the MAC0416 galaxies were calculated using the code Lephare of Arnouts \& Ilbert (2011), which fits stellar population synthesis models (Bruzual \& Charlot 2003) to the available photometry (Subaru $B, R$ and $z$-bands). This code is a simple $\chi^{2}$ minimization algorithm that finds the best match of templates for the given data. The limited number of filters results in a reduction of used templates. In particular, we do not fit templates with stellar ages of less than 1 Gyr and larger than 11 Gyr. This is a valid assumption since at $z=0.4$ the Universe is $\sim 4$ Gyr younger than today, and a considerable fraction of the stellar mass assembly is already completed. In addition, we kept the redshift fixed and also limited the number of extinction $E_{(B-V)}$ values $(0.1,0.3,0.5)$ to avoid over-fitting. We are confident in the robustness of the calculated masses since the $B-R$ color encompasses the redshifted $4000 \AA$ break and thus is sensitive to galaxy mass-to-light ratios (Kauffmann et al. 2003b). We list in Table A.1 the uncertainties on the stellar masses corresponding to the $68 \%$ confidence level on the mass, calculated by scanning the $\chi^{2}$ levels, while the redshift for each galaxy is kept fixed, but the other parameters (dust extinction, SFR, age, metallicity) are allowed to vary. 
To test the presence of any bias due to the limited broadband photometry, we produced synthetic B, Rc, and $z$-band magnitudes from Bruzual \& Charlot (2003) models with a range of stellar masses, ages, metallicities, star formation histories, and extinction values using the $E z G a l$ tool (Mancone \& Gonzalez 2012). These magnitudes were fed to Lephare and then the output was compared to the input values. Overall stellar masses are more resistant than other parameters, and show a scatter of about $0.1 \mathrm{dex}$. This additional error is generally smaller than the typical uncertainties reported in Table A.1.

As described in Maier et al. (2015), the stellar masses for zCOSMOS galaxies were derived by fiting photometric data points with the Bruzual \& Charlot (2003) synthetic stellar population models, and picking the best-fit parameters by minimizing the $\chi^{2}$ between observed and model fluxes. The stellar masses were obtained by integrating the SFR over the galaxy age, and correcting for the mass loss over the course of stellar evolution. Thus, the stellar masses of zCOSMOS galaxies are comparable to the stellar masses of MAC0416 galaxies, which are also based on SED fitting using Bruzual \& Charlot (2003) models. We converted the masses from a Chabrier (2003) IMF to a Salpeter (1955) IMF (as used in this paper) by multiplying the Chabrier (2003) IMF masses by 1.7. This factor of 1.7 was found by Pozzetti et al. (2007) to be a systematic median offset, with a very small dispersion, in the masses derived with the two different IMFs; it is rather constant for a wide range of SFHs. Regarding the consistency of SDSS stellar masses with stellar masses derived from SED fitting, we note that Moustakas et al. (2013) showed the comparison between the two methods of estimating stellar masses indicating that they are consistent within the uncertainties.

\subsection{Morphologies}

The decomposition of galaxies into their main stellar components is done by analyzing the light distribution with the sum of two Sérsic profiles with different indices (e.g., a DeVaucouleurs bulge and an exponential disk). To quantify stellar structures of MAC0416 galaxies, we measure parameters such as Sérsic indices, sizes, and luminosities for the entire galaxy, and for bulges and disks separately for all available bands (Subaru $B, R$, and $z$ ) with the new galaxy profile model fitting software Galapagos-2 developed within the MegaMorph project (Häußler et al. 2013). It performs two-dimensional modeling of the galaxy flux assuming predefined light distributions for all bands available simultaneously. Galapagos- 2 is constructed in a way to efficiently handle modern large multiwavelength imaging surveys and is thus ideally suited to analyze the CLASH data. It utilizes GALFIT-M (Bamford et al. 2011), a recently developed multiwavelength version of the two-dimensional fitting algorithm GALFIT (Peng et al. 2010b). This novel approach was successfully tested on both ground-based and simulated data (Häußler et al. 2013; Vika et al. 2014) and leads to an increase in the stability and accuracy of measured parameters down to fainter limits in comparison to the widely used single-band fits. This is especially important for the measurements of groundbased Subaru imaging for this study.

To ensure reliable morphologies, we compare our Subarubased classification step by step with HST high-resolution CLASH data. However, we note that none of the galaxies in our Zgals sample is in the central part of the cluster covered by HST. We therefore analyze an independent sample of about 100 overlapping galaxies of the central cluster region and find that $10 \%(15 \%)$ of the galaxies are misclassified using bulge-to-disk decompositions (single Sérsic fits). Whether we classify based on a single Sérsic fit or bulge-to-disk decomposition only changes the outcome in $5 \%$ of the cases (based on measurements of 820 galaxies in CLASH clusters covered with Subaru; Kuchner et al., in prep.). With F-statistics following Simard et al. (2011) we compare the residual images of oneand two-component fits to decide which model better represents the light distribution of a particular galaxy. To classify the cluster galaxies, we expand the decision tree of Neichel et al. (2008) (also adopted by others, for example Nantais et al. 2013) to our needs and include the new capabilities of Galapagos- 2 with varying sets of bulge and disk profiles, as well as color information and visual classifications. This leads to four distinct classes: compact, peculiar, disk-like, and objects with a smooth regular light distribution.

Specifically, we follow three main steps for the morphological analysis of the Zgals sample: i) we perform surface brightness profile measurements with Galapagos-2 using three Subaru-bands for $B / T$ and Sersic index $n$; ii) we construct RGB color maps for every GALFIT-M stamp; and iii) we label each galaxy morphologically based on visual inspection in both the individual images and the color map. The classification starts with dividing the sample of star-forming galaxies into three broad $B / T$ bins $(B / T<0.5,0.5<B / T<0.8$, and $B / T>0.8)$ with $\sim 3 \%$ incorrect measurements in the general comparison to HST in the overlap region. Each stamp is then visually inspected for spiral features, color gradient, and offsetting nuclei (see Nantais et al. 2013, for details). Owing to the low resolution of the Subaru imaging, only the most striking spiral features are seen and a smooth appearance is common. Therefore, we call high $B / T$ objects without any visible features smooth. It should be remembered, however, that these are still star-forming galaxies with a prominent bulge and no obvious signs of spiral structures. Galaxies with effective radii $r_{\mathrm{e}}<3 \mathrm{kpc}$ (corresponding to less than $0.55 \operatorname{arcsec}$ at $z \sim 0.4$ ) are classified as compact. Low $B / T$ (or low Sérsic index) galaxies and those with spiral features are classified as disk galaxies, while galaxies with visible disturbances in their stellar structure are called peculiar.

\subsection{SFRs}

One of the most reliable and well calibrated SFR indicators is the $\mathrm{H} \alpha$ EL. The conversion of $\mathrm{H} \alpha$ luminosity to SFR requires several steps. To correct for slit losses for Zgals MAC0416 cluster galaxies we convolve each VIMOS spectrum with the Subaru Cousins $R$-band filter and then compare this magnitude with the Subaru $R$-band magnitude of the respective galaxy. The difference between the two magnitudes gives the aperture correction factor for each spectrum. This correction assumes that the $\mathrm{H} \alpha$ line flux and the $R$-band continuum suffer equal slit losses and that the $\mathrm{EW}$ of $\mathrm{H} \alpha$ is constant throughout the entire galaxy. Thus revised $\mathrm{H} \alpha$ line luminosities $\mathrm{L}(\mathrm{H} \alpha)$ are corrected for extinction based on the Balmer decrement and then transformed into SFRs by applying the Kennicutt (1998) conversion: $\operatorname{SFR}\left(M_{\odot} \mathrm{yr}^{-1}\right)=7.9 \times 10^{-42} L(\mathrm{H} \alpha) \mathrm{ergs} / \mathrm{s}$.

The SFRs of the zCOSMOS sample at $z \sim 0.4$ and of SDSS galaxies are also computed from extinction corrected $\mathrm{H} \alpha$ luminosities. For the zCOSMOS galaxies an aperture correction based on the observed I-band magnitude was applied to each VIMOS spectrum to take into account slit losses, as described in Maier et al. (2009). The SDSS SFRs were corrected for fiber losses using the aperture corrections given by Brinchmann et al. (2004), corrections which work for $z>0.04$, as demonstrated by Kewley et al. (2005). 


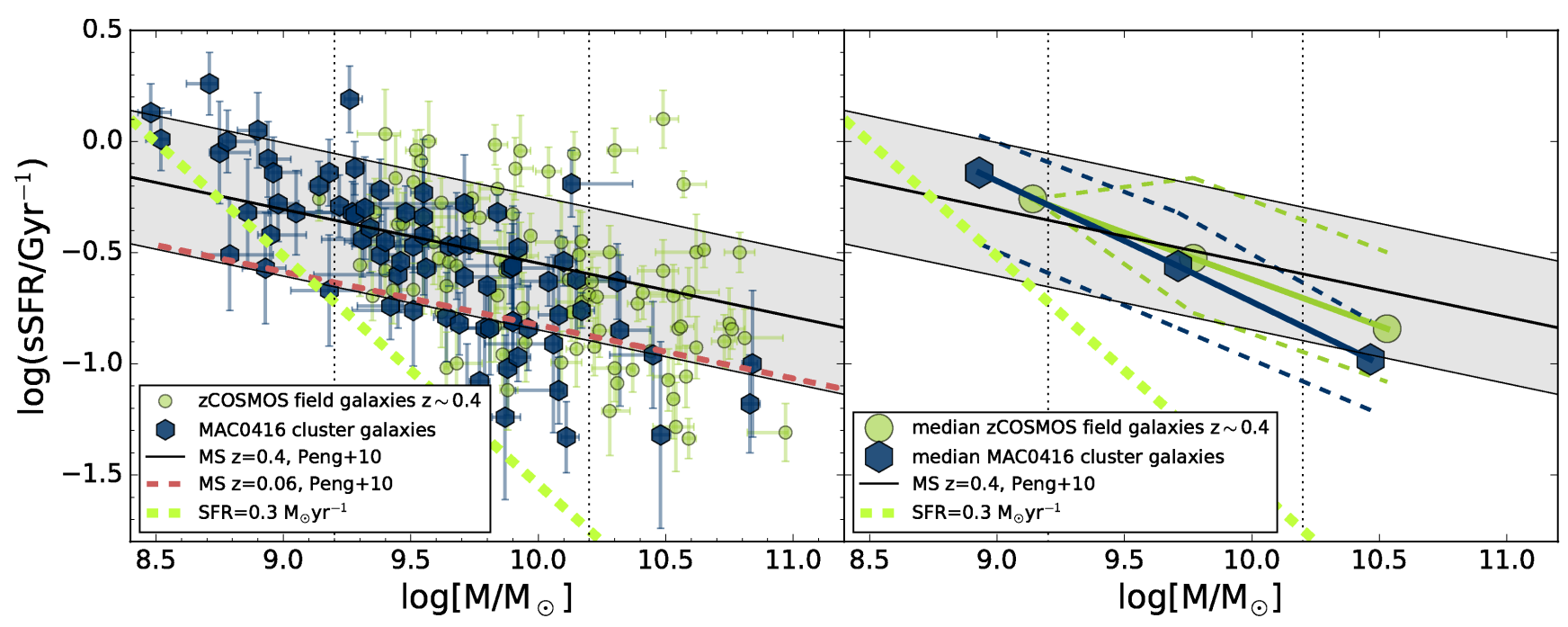

Fig. 3. Left panel: mass-sSFR relation for MAC0416 cluster galaxies (blue symbols) and zCOSMOS field galaxies (green) at $z \sim 0.4$. The oblique solid thick black line shows the MS at $z \sim 0.4$ and its dispersion (indicated by the gray area), and the dashed red line depicts the local SDSS MS, using Eq. (1) in Peng et al. (2010a) for $z \sim 0.4$ and $z \sim 0.06$, respectively. Right panel: median values of the mass-sSFR relations at $z \sim 0.4$ are shown as blue (cluster) and green (field) solid lines and big symbols, while the corresponding dashed oblique lines enclose $68 \%$ of the data. The dashed thick light green line shows the completeness limit of our Zgals cluster sample, $S F R \sim 0.3 M_{\odot} / \mathrm{yr}$.

\section{Results: mass-metallicity and mass-sSFR relations in field and cluster}

We discuss several aspects of galaxy evolution in different environments, each presented in the framework of the relations between mass and sSFR (Sect.4.1) and mass and metallicity (Sect. 4.2). We present evolutionary effects between redshifts $z \sim 0.4$ and $z \sim 0$, establishing the MZR at $z \sim 0.4$. We then subdivide the samples to study effects of the cluster and field environments. Finally, we include a more detailed investigation of morphology dependencies of each relation. For a meaningful investigation and considering our relatively small sample of targets, we divide the galaxies into three (physically motivated) mass-bins:

i) Galaxies with $M_{\text {high }}\left(\log \left(M / M_{\odot}\right)>10.2\right)$ lie above a threshold invoked by chemical downsizing in the mass-metallicity plane, manifested in the flattening of the MZR at high stellar masses (cf. Maier et al. 2015). Furthermore, this highmass regime is controlled by early-type and bulge-dominated galaxies, which explains the low number statistics for our cluster star-forming galaxies (see also Fig. 1).

ii) The intermediate-mass bin $M_{\text {med }}\left(9.2<\log \left(M / M_{\odot}\right)<10.2\right)$ is well populated by both field and cluster galaxies.

iii) Galaxies below $\log \left(M / M_{\odot}\right) \sim 9.2$, a stellar mass regime that we call $M_{\text {low }}$, will be considered low-mass objects. We do not have comparison galaxies in the field sample for this mass range owing to zCOSMOS-bright selection criteria.

\subsection{Mass-sSFR relation at $z \sim 0.4$}

The sSFR has been found to be a tight but weak function of mass at all epochs up to $z \sim 2$ for most star-forming galaxies (main sequence, $M S$ ), and to evolve with time increasing by a factor of $2-3$ to $z \sim 0.4$ compared to $z \sim 0$ (e.g., Noeske et al. 2007; Elbaz et al. 2007). Peng et al. (2010a) derived a formula for the evolution of the sSFR as a function of mass and time that we use to calculate the mean $\operatorname{sSFR}(M)$ at $z \sim 0.4$. For this, we assume a dependence of sSFR on mass as observed for local SDSS galaxies and revised by Renzini \& Peng (2015), $s S F R \propto M^{\beta}$ with $\beta=-0.24$ (Fig. 3, oblique solid thick black line). The local MS relation was found to have a dispersion of a factor of 0.3 dex about the mean relation (e.g., Salim et al. 2007; Elbaz et al. 2007; Peng et al. 2010a), indicated by the gray area around the mean MS relation at $z \sim 0.4$ in Fig. 3 .

The distribution in the mass-sSFR plane of our sample of field and cluster galaxies at $z \sim 0.4$ is comparable (Fig. 3), in particular in the stellar mass bin $M_{\text {med }}$ where the Zgals sample has a uniform coverage with respect to the parent sample (see Fig. 1). The median values of the two samples in this mass range and the scatter of the data points are also consistent with the mean MS relation and scatter predicted by Peng et al. (2010a) at $z \sim 0.4$. In the lower mass bin $M_{\text {low }}$ there are no field galaxies from zCOSMOS since the zCOSMOS-bright sample was selected to have $I<22.5$, so we cannot study environmental effects on SFRs for lower masses. In contrast, in the highest mass bin $M_{\text {high }}$ there are only six cluster galaxies in our sample, most of them showing lower sSFRs than typical MS galaxies at these redshifts.

Investigating in detail the morphological distribution in our sample of cluster galaxies, a segregation is revealed between bulge-dominated on the one hand and disk and peculiar galaxies on the other (Fig. 4). We measure a difference between the populations by comparing deviations of the median residuals of the fits. We find that cluster galaxies with prominent bulges lie systematically lower $(>1 \sigma)$ than the disk star-forming galaxies that are closely compatible with the MS. Stars in galactic bulges at $z \sim 0.4$ are dominated by an old, red, and passive population (Pérez et al. 2013), accounting little for the overall ongoing star formation of the galaxy. As a consequence, our galaxies in the smooth category, which have rather high $B / T$ values, are expected to display lower sSFRs. Peculiar galaxies on the other hand lie above the MS indicating an increase in current star formation with notable scatter. This type of galaxy is nearly impossible to distinguish through classifications based on single Sérsic $n$ and were therefore not captured in some previous studies.

Numerous observations describe an increase of bulgedominated galaxies in high-density regions, such as clusters of galaxies (e.g., Dressler 1980; Kormendy et al. 2009). The observed slope and scatter of the mass-sSFR relation reflect the 

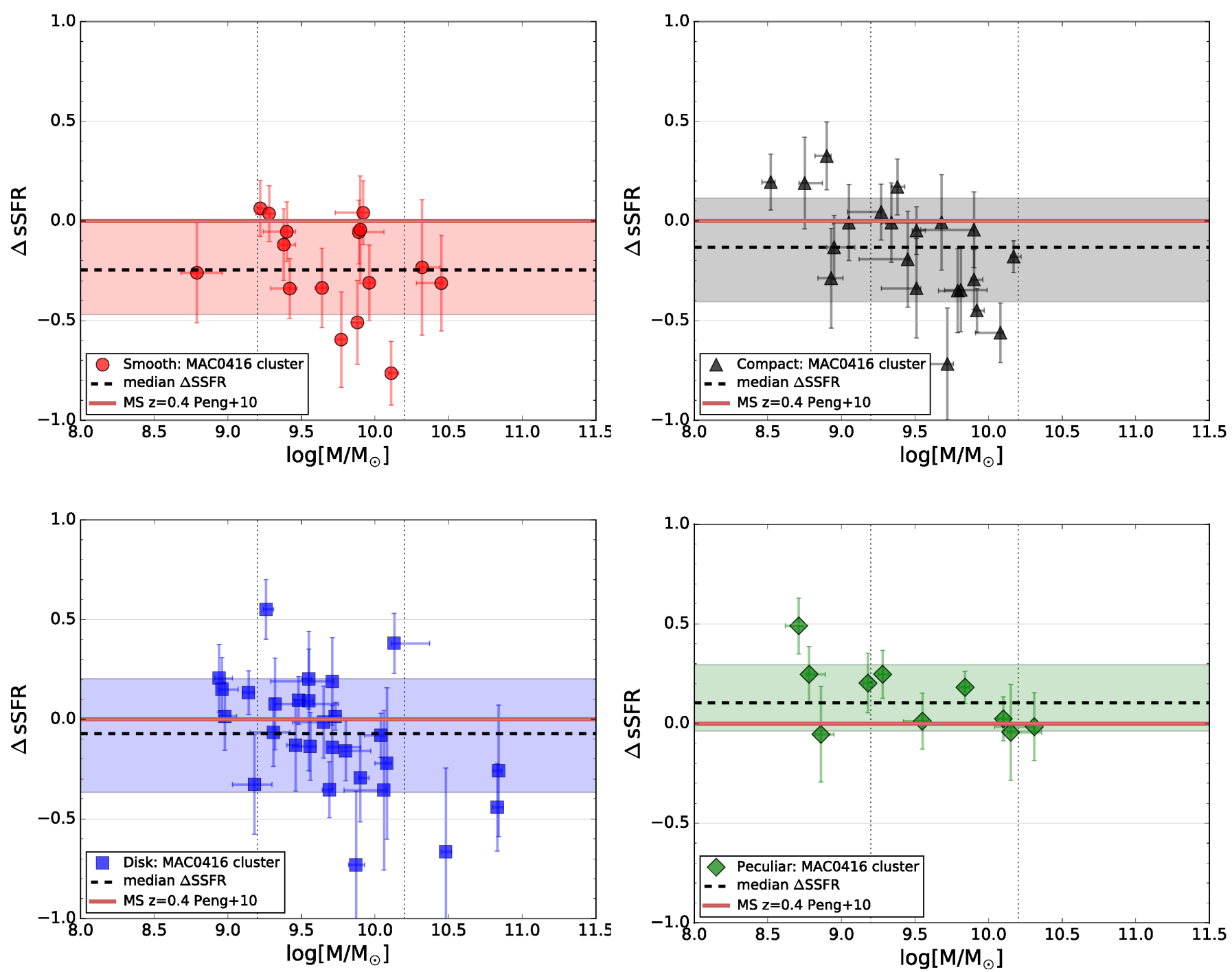

Fig. 4. Offsets of cluster galaxies with different morphologies from the MS at $z \sim 0.4$. Blue squares are disks, red circles smooth, black compact, and green irregulars. The red solid lines show the MS at $z \sim 0.4$ predicted by Peng et al. (2010a). The shaded area is the $1 \sigma$ scatter of the respective morphological category, while the dashed thick black line is the median value.

increase in bulge-mass fractions in higher mass galaxies and therefore a diversity in SFHs (Abramson et al. 2014). Our finding is in compliance with Whitaker et al. (2015), who link the scatter and slope of the MS to galaxy structures using single Sérsic measurements as a proxy for a dominant bulge or disk. For our work, we perform galaxy decompositions and therefore directly measure the presence and strength of bulges.

We see indications of the same trend of lower sSFRs in $\log \left(M / M_{\odot}\right)<10.2$ compact galaxies. However, decompositions into bulges and disks become less reliable for these small galaxies in ground-based imaging data (as revealed by our Subaru HST comparisons). Therefore, we can only speculate about the presence of a bulge in compact galaxies. In the local Universe, however, Ellison et al. (2008) have demonstrated a potential link between galaxy sizes and bulge fractions. They found that galaxies with smaller half light radii $\left(r_{\mathrm{e}}<3 \mathrm{kpc}\right)$ have higher $B / T$ ratios $(B / T>0.4)$, which supports our hypothesis. Figure 4 shows similar effects of lower sSFRs for smooth galaxies (bulgedominated) and to some extent also for compact galaxies on the one hand, and of higher sSFRs for disk and peculiar types (low bulge fractions) on the other hand.

\section{2. $M Z R$ at $Z \sim 0.4$ in field and cluster}

Figure 5 shows the MZR of our Zgals sample of 76 MAC0416 $z \sim 0.4$ cluster members (blue symbols) and 93 zCOSMOS field galaxies (green symbols) compared to SDSS (gray area). All metallicities were computed with the same method described in Sect. 3.3. For the matched SDSS sample constructed as described in Sect. 2.3, we indicate 16th and 84th percentiles, and the medians (50th percentiles) of the distribution of $\mathrm{O} / \mathrm{H}$ values in the respective mass bin as solid lines and gray area. We note that the mean SDSS O/H values in the respective mass bins differ from the median $\mathrm{O} / \mathrm{Hs}$ by less than $0.01 \mathrm{dex}$. We also extrapolate the MZR of the matched SDSS sample to lower stellar masses by assuming that the slope of the low-mass MZR stays constant for $\log \left(M / M_{\odot}\right)<9.2$. While both the local and distant sample of field galaxies have stellar masses $\log M / M_{\odot}>9.2$, several Zgals cluster galaxies extend to lower masses $\log \left(M / M_{\odot}\right) \sim 8.5$ thanks to the deep spectroscopy of CLASH-VLT.

The polynomial fits (third degree) to the data points (blue and green thick lines in the left panel of Fig. 5) and also the median values (right panel) are very similar for cluster and field galaxies of intermediate masses. We determine an evolution of $\sim 0.1 \mathrm{dex}$ 


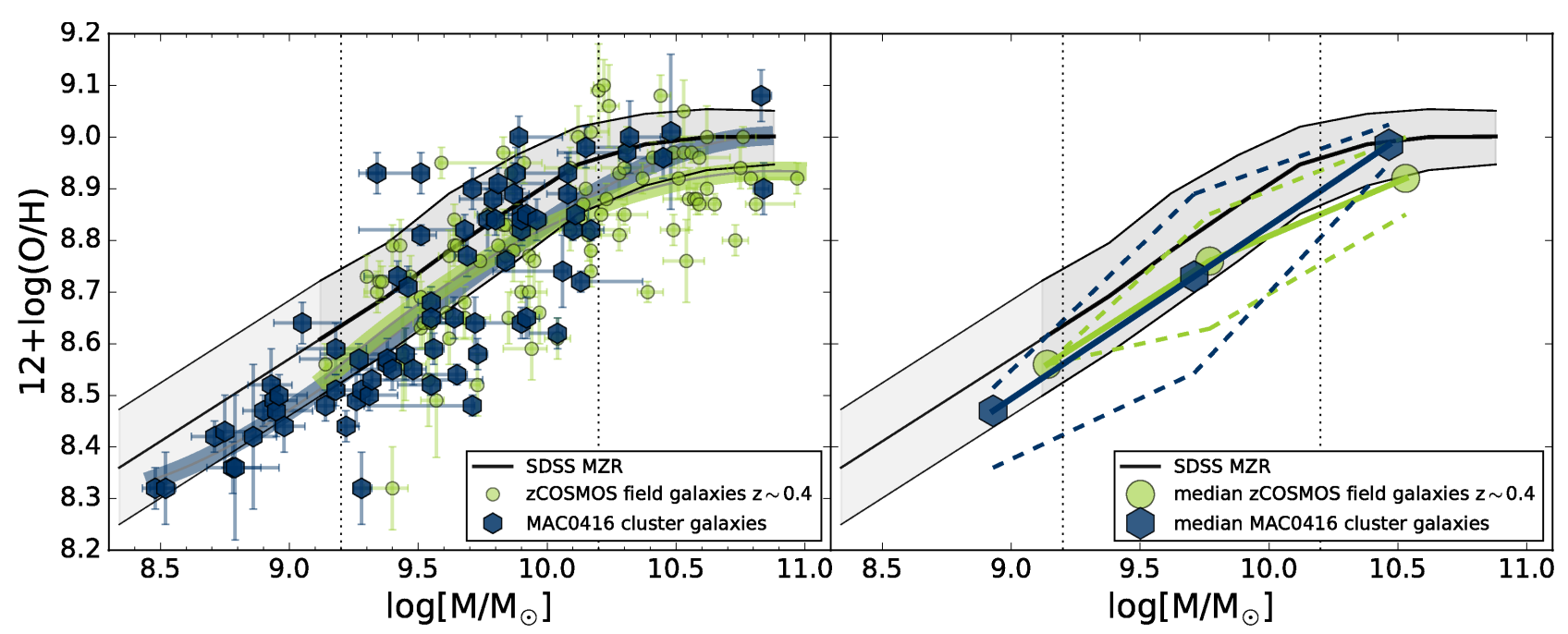

Fig. 5. Left panel: MZR for 76 MAC0416 cluster members (Zgals sample, blue symbols) and 93 field galaxies (green symbols) at $z \sim 0.4$. Polynomial fits to the data points (blue and green thick lines) show that the MZRs for cluster and field galaxies are similar at intermediate masses $M_{\text {med }}$, where the Zgals sample has a uniform coverage with respect to the parent sample (see Fig. 1). Right panel: median values of MZR at $z \sim 0.4$ are shown in blue (cluster) and green (field), with the corresponding dashed oblique lines enclosing $68 \%$ of the data. In both panels we extrapolated the MZR of the SDSS sample to lower stellar masses by assuming that the slope of the low-mass MZR stays $\operatorname{constant}$ for $\log \left(M / M_{\odot}\right)<9.2$.

on average for these $M_{\text {med }}$ galaxies. This offset for field galaxies towards lower metallicities is in agreement with the value given by Pérez-Montero et al. (2013). From their Table 3 of their zCOSMOS sample at similar redshifts, we infer an evolution of $\sim 0.09$ dex.

At the highest masses $M_{\text {high }}$ most of the zCOSMOS field galaxies have lower $\mathrm{O} / \mathrm{Hs}$ than the local ones, while the highmass cluster galaxies (albeit with only a few measurements) exhibit higher $\mathrm{O} / \mathrm{H}$ values, comparable to SDSS metallicities. This may indicate that the "chemical downsizing" effect described in Maier et al. (2015) regarding the time evolution of the mass threshold below which one finds low metallicities may have a different mass/time dependence in field and cluster environment. This indicative interpretation has to be verified with a larger sample of metallicity measurements for cluster starforming $M_{\text {high }}$ galaxies.

In their environmental study of central and satellite SDSS galaxies, Pasquali et al. (2012) report a small metallicity increase of $0.06 \mathrm{dex}$ at $\log M / M_{\odot} \sim 8.5$, which decreases to $0.004 \mathrm{dex}$ at $\log M / M_{\odot}>9.3$, for satellite galaxies in comparison to central galaxies of the same stellar mass. At higher redshifts we do not have the benefit of such a large database of metallicity measurements and restrictions in mass ranges or smaller samples like ours cannot reproduce this accuracy.

In their publication, Pasquali et al. (2012) speculate whether the slight increase of the MZR at lower masses found in satellite galaxies can be explained through strangulation scenarios. Strangulation prevents satellite galaxies from accreting new, lowmetallicity gas which would otherwise dilute their interstellar medium. We will explore this scenario further in Sect. 5.1 when we discuss the $Z(M, S F R)$ of cluster galaxies.

In a numerical work of environmental effects on metallicities, Davé et al. (2011) used cosmological hydrodynamic simulations and compared the MZR of model galaxies subdivided by local galaxy density. They found that model galaxies in highdensity regions lie above the mean MZR by $\sim 0.05 \mathrm{dex}$; however, the differences disappear at the massive end in all models. The general trend of both studies, numerical works and local observations, agree with our findings: the MZRs of cluster and field galaxies differ only marginally.

Several studies in the local Universe found a correlation between gas-phase metallicites and galaxy morphology, such that more bulge-dominated galaxies are more metal rich (e.g., Vila-Costas et al. 1992; Zaritsky 1993; Zaritsky et al. 1994). Similar trends that connect samples of high concentration galaxies (bulge-dominated) to higher fractions of solar metallicities in comparison to low-concentration samples (more disk-like) and lower metallicities were reported by Pasquali et al. (2012). Morphologies in our sample are uniformly distributed over stellar masses and colors within the blue cloud, especially in our intermediate-mass bin $M_{\text {med }}$, and any trends found are therefore unlikely to result purely from the strong correlations between luminosity, color, and morphology. The chemical evolution of galaxies is connected to the $\mathrm{SFH}$, and is also influenced by the environment through gas interchange with the surroundings. We therefore expect trends to be intimately linked to the observed scatter of the MS and differences in sSFRs. Figure 6 shows the median offsets to the local SDSS MZR for Zgals MAC0416 galaxies, divided by their morphologies. Just like in the masssSFR relation, we find different behaviors for smooth/compact vs. disk/peculiar types. In agreement with predictions from the FMR, the smooth and compact galaxies that have lower sSFRs display higher metallicities with mean values only slightly below, and in some cases comparable to (within $1 \sigma$ ), the mean local MZR. In contrast, disk-dominated and peculiar objects (with higher sSFR) exhibit metallicities well below the local MZR.

We note that, as discussed in Sect. 4.1, smooth and compact galaxies have higher bulge fractions than disk-dominated and peculiar objects. Simulations have shown that bulges appear to stabilize against inflows of metal-poor gas and nuclear starbursts driving galactic outflows (e.g., Mihos \& Hernquist 1994, 1996; Cox et al. 2008). Bulge-dominated galaxies like the smooth galaxies in our Zgals sample therefore possibly retain their metal-enriched gas more easily. For the other types, like disk and peculiar Zgals galaxies, inflowing gas may be funneled more efficiently to their centers with low bulge fractions. 

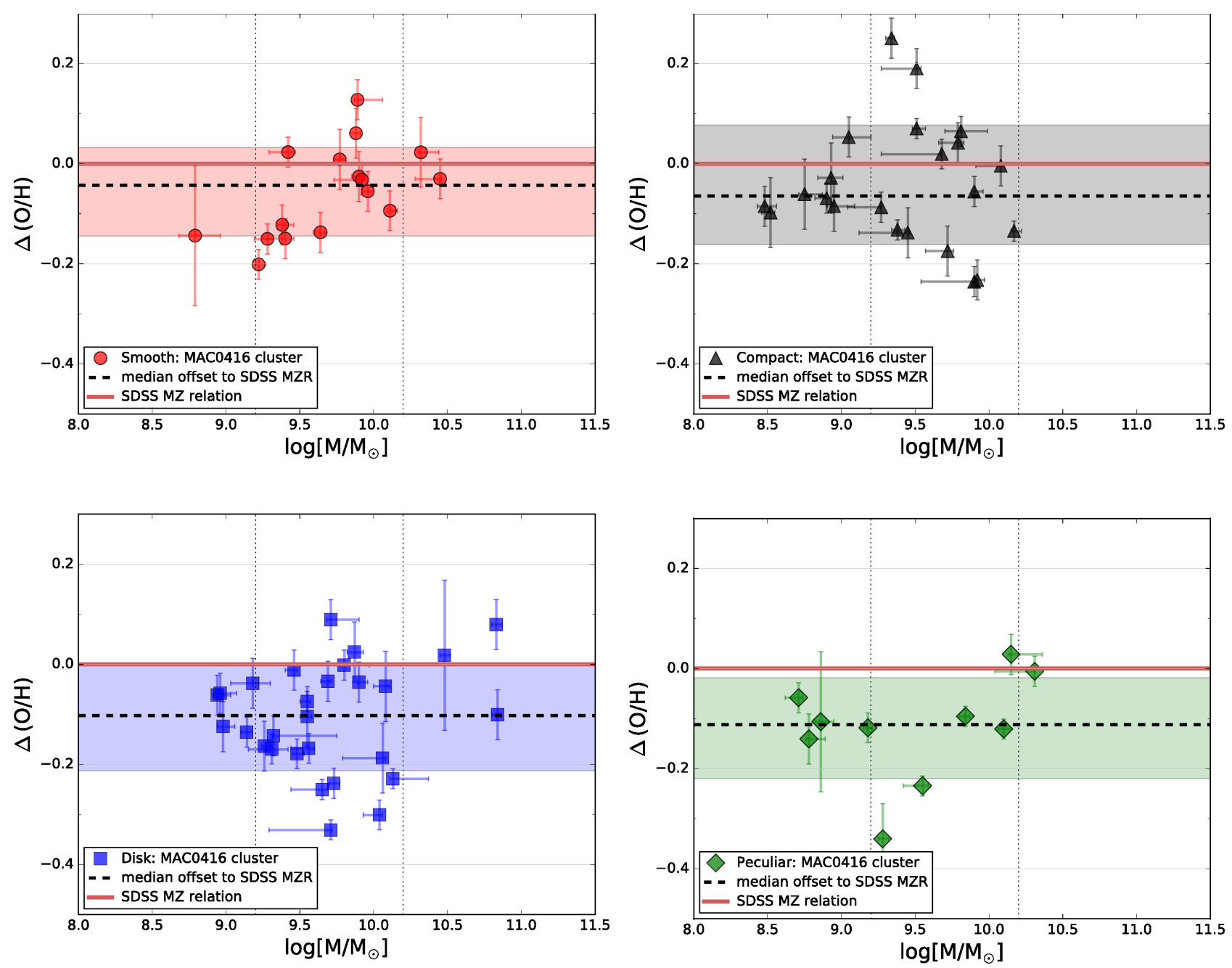

Fig. 6. Median offsets to the local relation for Zgals MAC0416 galaxies, divided by their morphologies. We find different behaviors for smooth/compact vs. disk/peculiar types with respect to the local relation. While for the latter we measure $\mathrm{O} / \mathrm{H}$ abundances that are lower than the local relation, those galaxies with more prominent bulges and compact galaxies are more enhanced in gas metallicities.

\section{Discussion: environmental effects and evidence for starvation}

The location of galaxies in projected phase-space (cluster-centric radius vs. line-of-sight velocity relative to the cluster redshift) provides valuable information on their accretion history: on average, infalling galaxies are spatially separated from virialized galaxies. Simulations of mass assembly around clusters have helped to identify these regions in phase-space. Stacked caustic (isodensity profile) plots of galaxies orbiting the 30 most massive clusters in the Millenium simulation demonstrate how galaxies of different accretion histories populate different areas in a characteristic trumpet shaped caustic profile (Haines et al. 2015). Recently accreted galaxies (with, in general, higher lineof-sight velocities) are located outside these caustics and are thus well separated from the dense cluster core where galaxies are more likely to have been accreted at earlier times.

For our analysis, we define the rest-frame velocity as $v_{\mathrm{rf}}=$ $c\left(z-z_{\text {mean }}\right) /\left(1+z_{\text {mean }}\right)$, where $z_{\text {mean }}=0.3972$ is the mean cluster redshift, and we depict $R$ vs. $\left|v_{\mathrm{rf}}\right|$ in Fig. 7, with $\mathrm{R}$ being the projected radial distance from the cluster center. To separate mass and environmental effects, we concentrate on a limited range in stellar mass and consider only 53 MAC0416 cluster galaxies with $M_{\text {med }}\left(9.2<\log \left(M / M_{\odot}\right)<10.2\right)$. The dashed magenta line in Fig. 7 indicates the caustic determined by B16, separating the regions in phase-space of recently accreted and infalling galaxies (outside caustics) from the region of the galaxies accreted longer ago and a possibly virialized population (using the nomenclature of Jaffé et al. 2015; see also Haines et al. 2015). For simplicity, we call the two classes "infalling" (above and to the right of the magenta dashed line, star symbols) and "accreted" (filled circles).

We note that only 5 out of 18 infalling $M_{\text {med }}$ galaxies (28\%) have higher $\mathrm{O} / \mathrm{Hs}$ consistent with the local MZR, while this fraction is higher for accreted $M_{\text {med }}$ objects, namely 22 out of 35 galaxies (63\%). As discussed and shown in the following, infalling galaxies with lower metallicities than the local MZR (blue star symbols, representing $72 \%$ of the infalling population) can increase their $\mathrm{O} / \mathrm{Hs}$ by $\sim 0.2 \mathrm{dex}$ in $t_{\text {enrich }} \sim 1$ Gyr reaching the local MZR while they move towards the central region of the cluster. This enrichment time is shorter than the crossing time $t_{\text {crossing }}=R_{200} / \sigma$ of the cluster, which is about $2 \mathrm{Gyr}$, and also smaller than the 2-3 Gyr needed by galaxies in the infalling region to reach the central, denser part of the cluster, as derived by Haines et al. (2015). This makes a strangulation scenario a plausible mechanism, in which the gas metallicity increases because the reservoir of pristine gas is depleted when the galaxies enter the cluster. We explore additional evidence for this scenario in the following sections. 


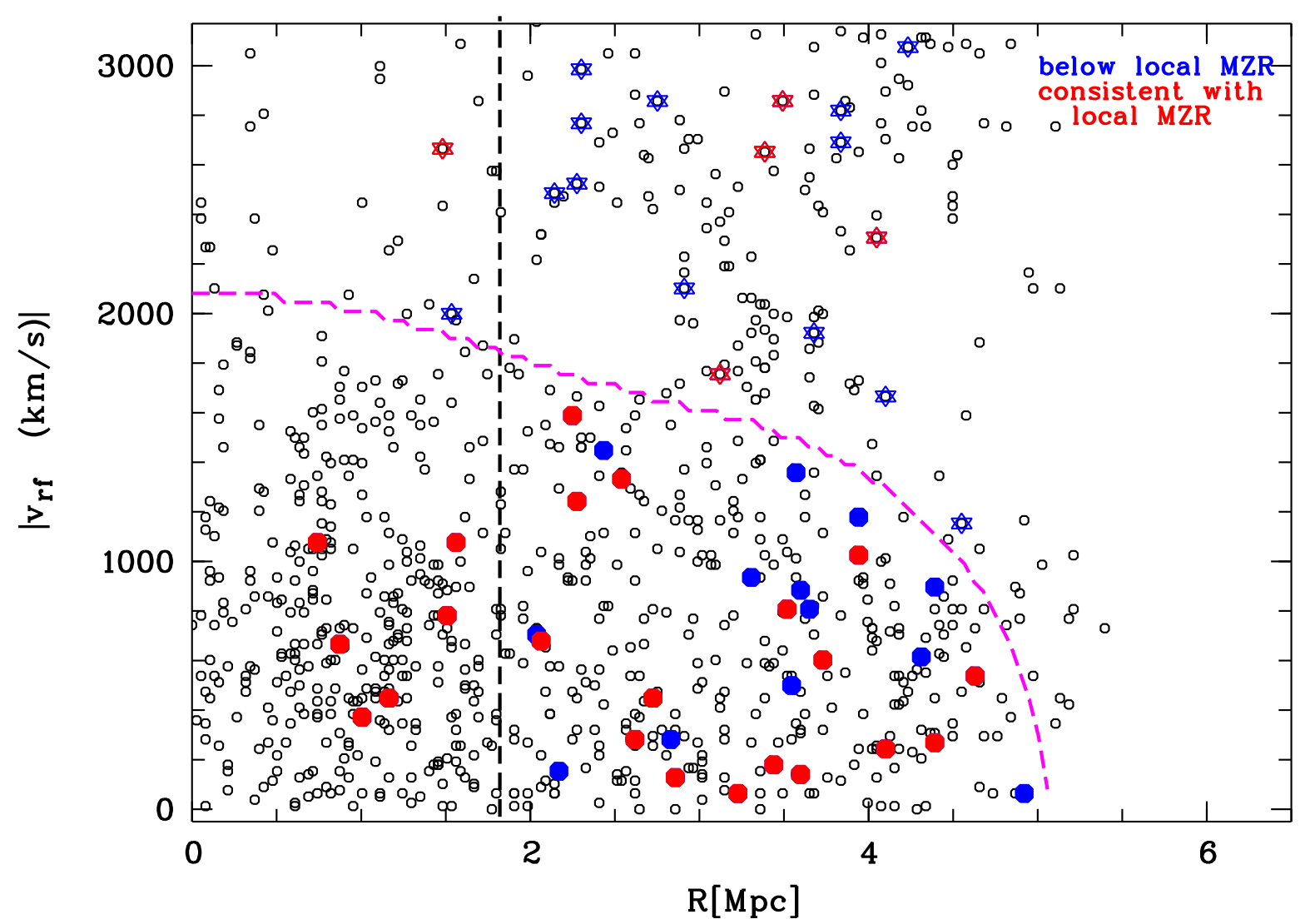

Fig. 7. Phase-space diagram based on all MAC0416 spectroscopic members (black open circles). Our study concentrates on the objects for which we were able to measure metallicities (Zgals sample). Restricting ourselves to intermediate masses $9.2<\log \left(M / M_{\odot}\right)<10.2$ we depict high metallicity objects (consistent with the local MZR) as red symbols and galaxies with lower metallicities (below the local MZR) as blue symbols. The dashed vertical line indicates $R_{200} \sim 1.8 \mathrm{Mpc}$ of the cluster (as reported in B16); the dashed magenta line indicates the caustic determined by B16. The diagram enables us to divide the cluster galaxies into infalling objects (above and to the right of the magenta line, star symbols) and accreted objects (filled circles).

\subsection{The $Z(M, S F R)$ of cluster galaxies at $z \sim 0.4$}

In order to explain star formation as a second parameter in the MZR, some authors have ascribed an ad hoc inflow of gas as the driver responsible for the dilution of metallicity and increase in SFR (Mannucci et al. 2010; Davé et al. 2012; Dayal et al. 2013). A different explanation was put forward by Li13, who proposed a simple model of galaxy evolution in which the SFR is regulated by the mass of gas present in a galaxy, implying that $Z$ depends on both stellar mass and current SFR. They derived a $Z(M, \mathrm{SFR})$ relation that depends on the internal parameters describing the regulator system, specifically the star formation efficiency $\epsilon=S F R / M_{\text {gas }}$ and the mass-loading of any wind that drives gas out of the system, their $\lambda=$ outflow/SFR. The local SDSS data from Mannucci et al. (2010) can be reproduced with astrophysically plausible values of $\epsilon(M)$ and $\lambda(M)$ (see Eq. (40) in Li13 and Eq. (3) in Maier et al. 2014), $Z_{\mathrm{eq}}=$ $Z_{0}+y /\left(1+\lambda(1-R)^{-1}+\epsilon^{-1}((1+\beta-b) S F R / M-0.15)\right)$, where $Z_{\mathrm{eq}}$ is the equilibrium value of the metallicity, $Z_{0}$ is the metallicity of the infalling gas, $y$ the yield, and $R$ the returned fraction. The possible values of the different parameters $\epsilon, \lambda, y$, and $b$ are given in Table 1 of Li13. These analytic approximations have since been verified by more detailed calculations in Pipino et al. (2014).

Our aim is to challenge this model description of a fundamental relation in a dense cluster environment at higher redshift, i.e., we want to investigate whether the $Z(M, \mathrm{SFR})$ dependence is similar to the one found in the local Universe so that the FMR is still valid. To this purpose we calculate the expected $\mathrm{O} / \mathrm{H}$ values from the Li13 model for each galaxy individually with their respective stellar mass M and SFR. We then compare the predicted values with the measured ones and show the difference in Fig. 8. We find that $87 \%$ of the zCOSMOS field comparison galaxies have $\mathrm{O} / \mathrm{H}$ values that agree with the model expectations within 0.2 dex, the nominal maximum error in $\mathrm{Z}$ determination. We conclude therefore that the majority of zCOSMOS field galaxies at $z \sim 0.4$ (with $\log \left(M / M_{\odot}\right)>9.2$ ) obey the FMR. This is in accordance with Cresci et al. (2012) who used a small subsample of zCOSMOS $z \sim 0.4$ galaxies, albeit with a strong $\mathrm{S} / \mathrm{N}$ selection: $S / N>20$ and $S / N>8$ in $\mathrm{H} \beta$ flux for $\log \left(M / M_{\odot}\right)>10$ and $\log \left(M / M_{\odot}\right)<10$, respectively. They also considered the difference between measured $\mathrm{O} / \mathrm{H}$ and predicted $\mathrm{O} / \mathrm{H}$ measurements, but used the Mannucci et al. (2010) predictions instead of the Li13 ones to conclude that the FMR holds for field galaxies at $z \sim 0.4$.

To investigate the FMR for cluster members, we show in panel c of Fig. 8 the difference in $\mathrm{O} / \mathrm{H}$ measurements from predictions using both primordial and enriched inflowing gas. Eighty-eight percent of Zgals cluster galaxies with $\log \left(M / M_{\odot}\right)>$ 9.2 have $\mathrm{O} / \mathrm{H}$ values that are in accordance with the model expectations within $0.2 \mathrm{dex}$, similar to the behavior of zCOSMOS field galaxies. In contrast, lower mass $M_{\text {low }}$ cluster members deviate systematically from the FMR predictions. For the first time we can explore the $Z(M, \mathrm{SFR})$ of star-forming cluster galaxies at higher redshifts down to low masses $M_{\text {low }}\left(8.5<\log M / M_{\odot}<\right.$ 9.2) with CLASH-VLT. These $M_{\text {low }}$ cluster galaxies are not 


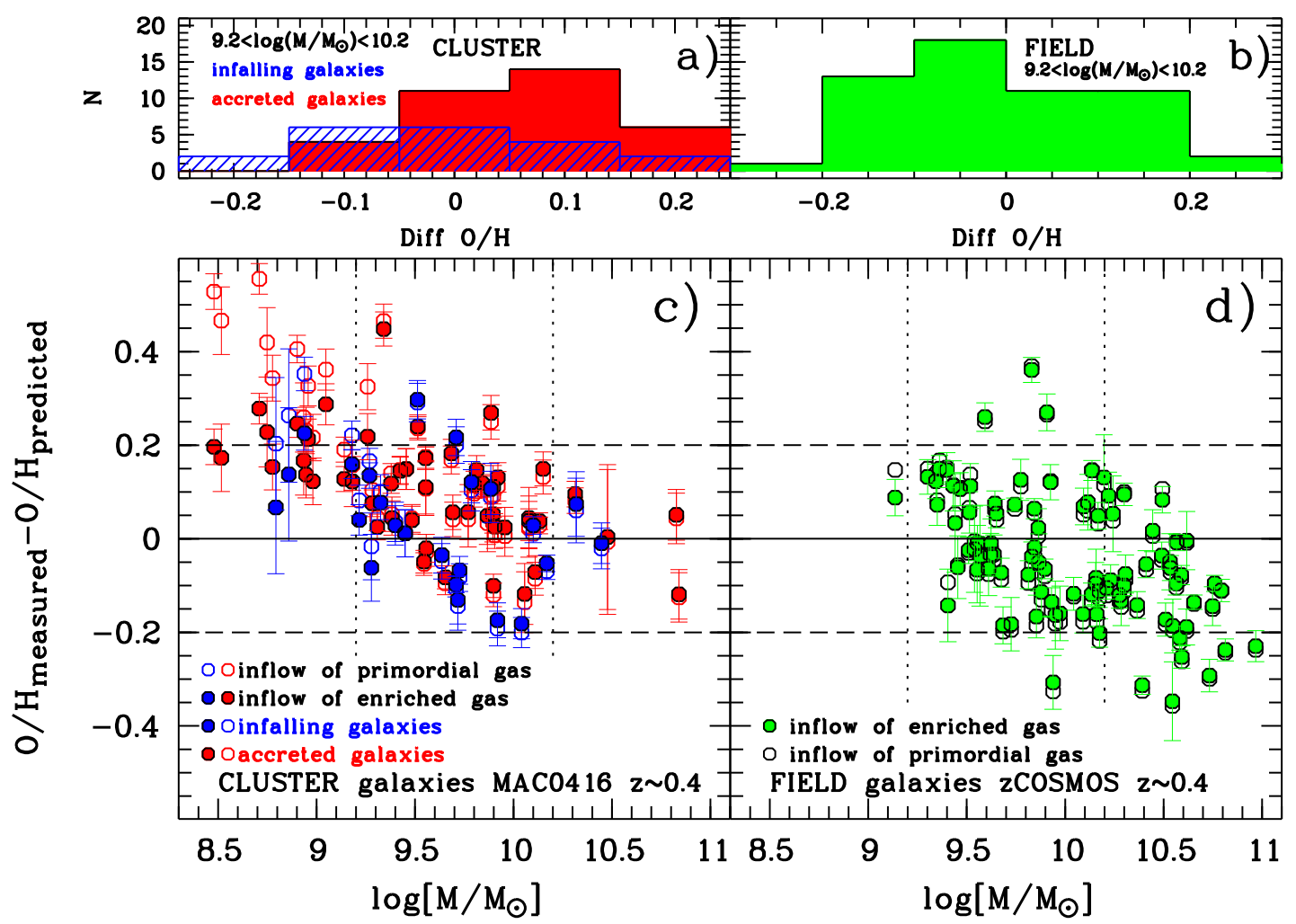

Fig. 8. Difference between the measured $\mathrm{O} / \mathrm{Hs}$ of $z \sim 0.4$ Zgals cluster galaxies (panel c)) and zCOSMOS field objects (panel d)) and the expected $\mathrm{O} / \mathrm{Hs}$ from the formulations of Li13 for different infall metallicities $Z_{0}$ relative to the yield $y, Z_{0} / y=0.1$ (enriched inflow, filled circles) and $Z_{0} / y=0$ (pristine inflow, open circles). In addition, in panel c)) the position of cluster galaxies in the phase-space diagram (see Fig. 7) is indicated in red (blue) for accreted (infalling) galaxies. The upper histograms (panels a) and b)) for $M_{\text {med }}$ objects show that in general the infalling cluster members (blue histogram) and field galaxies (panel b)) have lower metallicities, in quite good agreement with the FMR, unlike accreted galaxies (red histogram), which in general have higher metallicities than predicted by the FMR.

compatible with the FMR if primordial inflowing gas $\left(Z_{0}=0\right)$ is assumed (open circles), having metallicities two to three times higher than predicted. We note that most of these low-mass galaxies are accreted objects (except three infalling $M_{\text {low }}$ objects shown in blue). This suggests that galaxies of lower masses $M_{\text {low }}$ are more prone to environmental effects when they are accreted by the cluster owing to their lower gravitational potential. Better agreement with the FMR can be achieved with infall of enriched gas with metallicity $Z_{0} / y=0.1$ (filled symbols). This is in compliance with the predictions of Peng \& Maiolino (2014) that the inflowing gas should become progressively metal enriched $\left(Z_{0}\right.$ is higher) in dense regions, producing higher metallicities for cluster galaxies of low masses compared to field galaxies of similar masses. Peng \& Maiolino (2014) do not give a physical explanation how metal-enriched gas inflow can happen in the dense cluster environment. However, they offer an alternative scenario based on strangulation, in which the inflow of pristine gas is stopped, as a possible explanation for higher $\mathrm{O} / \mathrm{Hs}$ in lower mass objects. They do not elaborate this scenario further because they find, as we do, that the sSFRs are similar in different environments (see Fig. 3).

It should be noted that we call strangulation/starvation a scenario in which the supply of cold gas onto the galaxy disk is halted because the halo gas is stripped owing to external forces. In this case star formation can continue, using the gas available in the disk until it is completely used up. Therefore, similar sSFRs for star-forming cluster and field galaxies do not completely exclude strangulation as a plausible mechanism for the chemical enrichment effects we see in the cluster galaxies. In a context of a rich cluster, this scenario was first described by Larson et al. (1980), who used the removal of the gas reservoir due to external forces to explain the passive cluster galaxy population.

To explore the strangulation scenario, we divide the $M_{\text {med }}$ cluster sample into infalling and accreted objects according to their position in the phase-space diagram (Fig. 7) and depict them in blue and red in Fig. 8. The difference between measured and predicted $\mathrm{O} / \mathrm{Hs}$ for most of these $M_{\text {med }}$ cluster objects is very similar for the two scenarios with inflow of primordial gas (open circles) and enriched gas (filled circles): the difference between the values of the open and the respective filled circles for most $M_{\text {med }}$ galaxies is less than $\sim 0.02$ dex. Therefore, the histograms of $M_{\text {med }}$ galaxies shown in panel a) of Fig. 8 are very similar for the two scenarios of inflowing enriched or primordial gas. These histograms show that the $\mathrm{O} / \mathrm{H}$ distribution of $M_{\text {med }}$ infalling cluster galaxies is in good agreement with the FMR, while accreted galaxies have a distribution shifted to higher metallicities, in general higher than predicted by the Li13 model. This indicates that galaxies may increase their metallicities as a result of starvation after being accreted by the cluster. On the other hand, infalling galaxies are still star-forming, with their $\mathrm{O} / \mathrm{Hs}$ not yet influenced by environmental effects of the cluster, in general following the FMR and behaving like field galaxies (panel b of Fig. 8). In a recent paper, Peng et al. (2015) used the SDSS local sample and found that the stellar metallicity of satellite galaxies is slightly higher than that of central galaxies for stellar masses $\log \left(M / M_{\odot}\right)<10.2$ (value converted to a Salpeter IMF as used in this paper). They inferred that lower mass satellite galaxies are more prone to strangulation, implying that environmental effects are responsible for stopping the inflow of gas. We now find 

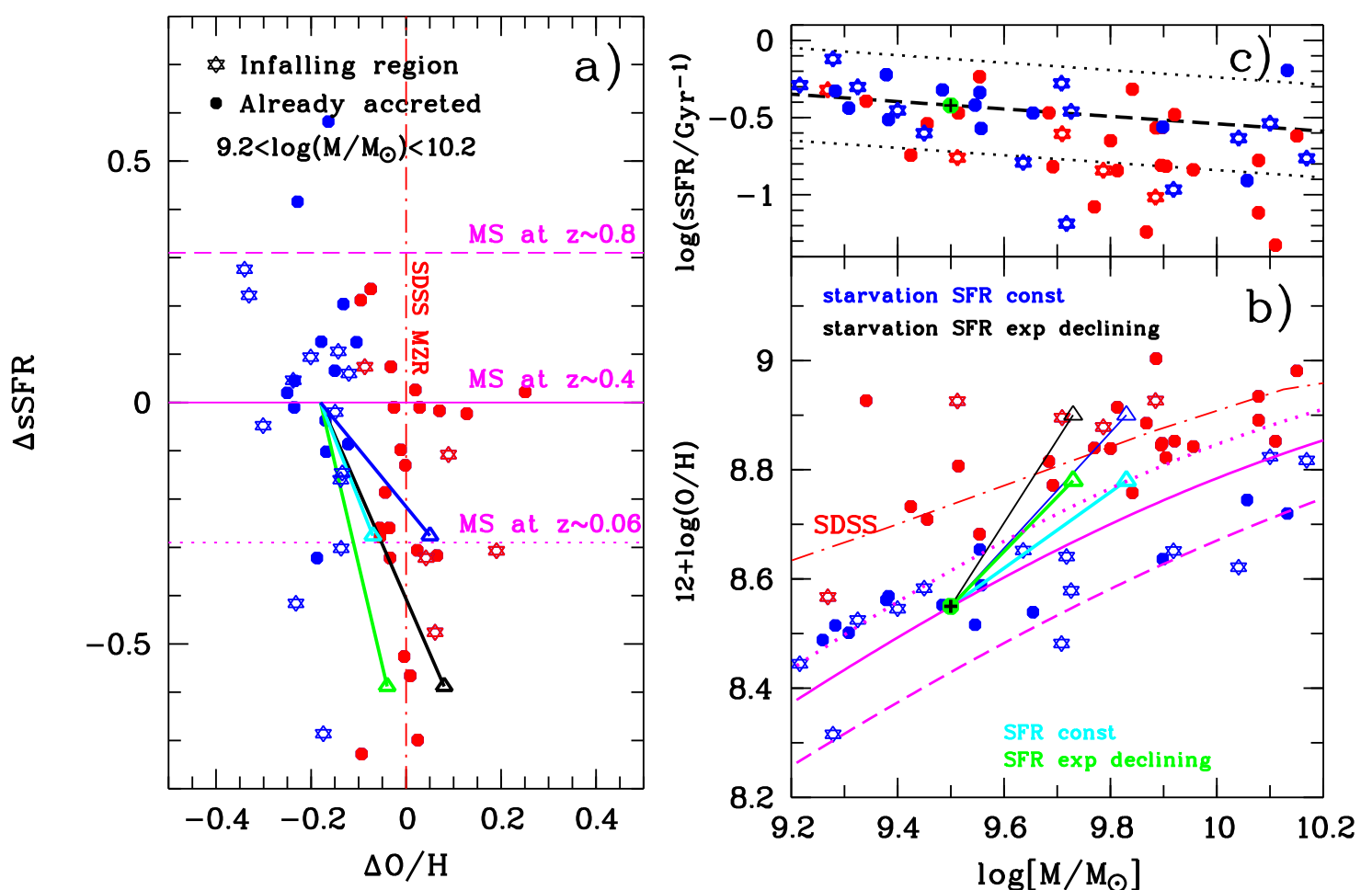

Fig. 9. Panel a): offsets $\Delta \mathrm{O} / \mathrm{H}$ to the local MZR vs. $\Delta$ SSFR to the MS at $z \sim 0.4$ for cluster galaxies with $\operatorname{masses} 9.2<\log \left(M / M_{\odot}\right)<10.2$ $\left(M_{\text {med }}\right)$. Filled circles (stars) indicate accreted (infalling) galaxies based on their position in the phase-space diagram (Fig. 7). Galaxies with O/Hs consistent with the local MZR are shown in red, other objects in blue. The MS mean values at $z \sim 0.8, z \sim 0.4$, and $z \sim 0.06$, based on the equation of the evolution of the sSFR from Peng et al. (2010a), are indicated by dashed, solid, and dotted magenta lines. The dash-dotted red line indicates the median local MZR of our matched SDSS sample (see also Fig. 5). Four tracks of a model galaxy start with $1.2 M_{\odot} / \mathrm{yr}$ and $\log \left(M_{\odot} / M_{\odot}\right)=9.5$. The model galaxy continues forming stars for $3 \mathrm{Gyr}$ with a constant SFR (cyan track) or exponentially declining SFR with a $\tau=3 \mathrm{Gyr}$ (green track), and the corresponding tracks are shown for a strangulation scenario with constant SFR (blue track) and exponentially declining SFR (black track). Panel b): MZR for $M_{\text {med }}$ cluster galaxies with data symbols and paths of model galaxies color-coded as in panel a). The magenta lines show the Li13 expectations of the $Z(M, S F R)$ for galaxies on the MS at different redshifts, with dashed, solid, and dotted lines corresponding to different $z$ like the magenta lines in the panel a). Panel c): the mass-sSFR relation for cluster galaxies color-coded in red for O/Hs consistent with the local MZR. Dashed and dotted lines show the median and dispersion of the mass-sSFR relation according to Peng et al. (2010a). The green filled circles with a black cross in panels b) and c) indicate the starting point of the toy models described in $\operatorname{Sect.} 5.2: \log \left(M_{\text {start }} / M_{\odot}\right)=9.5$, $S F R_{\text {start }}=1.2 M_{\odot} / \mathrm{yr}$, and $Z_{\text {start }}=8.55$. For the sake of visibility we do not plot error bars in Fig. 9; however, the error bars were already shown in the respective mass-sSFR (Fig. 3) and MZR plot (Fig. 5) for galaxies of all stellar masses.

additional signs of strangulation due to environment at $z \sim 0.4$ for galaxies with similar masses, $\log \left(M / M_{\odot}\right)<10.2$. In the next section, we discuss further evidence that strangulation is a plausible mechanism for explaining the metallicities of cluster galaxies.

\subsection{Co-evolution of SFR and Z}

To understand the combined SFR and metallicity evolution of galaxies, we consider four toy models of different SFHs: a constant SFR and primordial gas inflow; an exponentially declining SFR with a timescale $\tau$ of $3 \mathrm{Gyr}$ and inflow; and strangulation scenarios, i.e., without any inflow, for the two SFHs. We follow the evolution of the model galaxy in these four scenarios for $3 \mathrm{Gyr}$, which roughly corresponds to i) the time between the redshift of the MAC0416 cluster, $z \sim 0.4$, and the upper redshift range of the SDSS comparison sample, $z \sim 0.08$; and ii) the time span between $z \sim 0.8$ and $z \sim 0.4$. The four models allow us to follow i) the evolution of an infalling low metallicity galaxy (blue star symbols in Fig. 7) that will reach more inner regions of the cluster and increase its metallicity, as expected from the observed $\mathrm{O} / \mathrm{Hs}$ at $z \sim 0.4$ of most accreted cluster members, and ii) the evolution of a galaxy infalling into the cluster at $z \sim 0.8$ and becoming an accreted cluster galaxy when being observed at $z \sim 0.4$, likely becoming a higher metallicity object (red filled circles in Fig. 7). Thus, the paths of our model galaxies are assumed to start in a region populated by infalling low-metallicity galaxies, and are expected to end in the region of the mass-metallicity diagram populated by higher metallicity objects (with $\mathrm{O} / \mathrm{Hs}$ consistent with the local MZR, as indicated by the red dash-dotted line and red symbols in panel b of Fig. 9).

As discussed in the previous section, Fig. 8 demonstrates that the more massive $\left(\log \left(M / M_{\odot}\right)>9.2\right)$ field and infalling cluster galaxies at $z \sim 0.4$ are generally compatible with the $Z(M, S F R)$ relation as given by Li13 (their Eq. (40)). Since this equation, which we reproduced in Sect. 5.1, is by construction also applicable to local SDSS galaxies, we can assign a SFR value to every grid point in the MZR diagram. In the following we use $Z(\log M, S F R)$ to denote the metallicity $\mathrm{O} / \mathrm{H}$ as a function of stellar mass and SFR.

The starting point of the evolutionary tracks for the four model galaxies at $z=0.4$ (shown as a filled green circle with a black cross in panels b and c of Fig. 9) was derived by extrapolating the local MS to this redshift, following the evolution of the sSFR, derived in Peng et al. (2010a). Initial values for stellar mass and SFR are chosen as $\log \left(M_{\text {begin }} / M_{\odot}\right)=9.5$ and $S F R_{\text {begin }}=1.2 M_{\odot} /$ yr. Following considerations of the FMR (Li13), the model galaxy starts with an initial metallicity 
value of $Z_{\text {begin }}(9.5,1.2)=8.55$. These are typical (mean) $Z(\log M$, SFR) values for infalling, low metallicity, MS Zgals cluster galaxies with $9.2<\log \left(M / M_{\odot}\right)<9.8$ (blue star symbols in Fig. 9). On the other hand, the five cluster infalling galaxies with higher metallicities show, with one exception, lower sSFRs than the MS (red star symbols in panel c of Fig. 9). The lower sSFRs (and higher $\mathrm{O} / \mathrm{Hs}$ ) of these infalling galaxies could be due to pre-processing in the group environment (e.g., Zabludoff \& Mulchaey 1998) prior to arriving in the infall region of the cluster.

We follow the tracks of our model galaxies in the MZR and in the $\Delta s S F R-\triangle \mathrm{O} / \mathrm{H}$ diagrams. We chose $\triangle s S F R$ to be the offset to the MS at $z \sim 0.4$ (see Fig. 4), while $\Delta \mathrm{O} / \mathrm{H}$ is the offset between the measured $\mathrm{O} / \mathrm{H}$ and the local SDSS MZR value for $\mathrm{O} / \mathrm{H}$ at the respective mass (see Fig. 6). The sSFR offset from the MS at a given mass indicates the level of star formation compared to what is typical for a galaxy of that mass.

The magenta lines in panel b of Fig. 9 show the Li13 expectations of the $Z(M, S F R)$ for galaxies on the MS at different redshifts, with dashed, solid, and dotted lines corresponding to $z \sim 0.8, z \sim 0.4$ and $z \sim 0.06 \mathrm{MS}$ galaxies. A MS model galaxy with $Z_{\text {start }}(9.5,1.2)=8.55$ lies on the MZR of MS galaxies at $z \sim 0.4$ (solid magenta line). It forms stars for $3 \mathrm{Gyr}$ with a constant SFR and increases its stellar mass to $\log \left(M_{\text {end }} / M_{\odot}\right)=9.83$, and its metallicity to $Z_{\text {end }}(9.83,1.2)=8.78$ (cyan track). Although the SFR is constant, the sSFR of the model galaxy with constant SFR decreases because stellar mass is formed, reaching the expected MZR of MS galaxies at $z \sim 0.06$ (dotted magenta line in the panels a and b of Fig. 9). Thus, we note that a MS $z \sim 0.4$ model galaxy with constant SFR can reach the MS of SDSS galaxies without decreasing its SFR, but simply because it increases mass through constant SFR, which leads to a decrease in its SSFR.

The tracks for a model galaxy forming stars with an exponentially declining SFR on a timescale $\tau$ of 3 Gyr and having an inflow of gas are shown by the green tracks in panels a and b of Fig. 9. In this case $\log \left(M_{\text {end }} / M_{\odot}\right)=9.73, S F R_{\text {end }}=0.44 M_{\odot} / y r$, and the sSFR decreases after 3 Gyr by a larger amount than in the case of constant SFR (panel a), and the model galaxy reaches $\mathrm{Z}_{\text {end }}(9.73,0.44)=8.78$, the same metallicity as in the case of constant SFR (panel b). Any naively assumed difference in $Z_{\text {end }}$ (galaxies with lower $M_{\text {end }}$ should also have lower $Z_{\text {end }}$ ) cancels out because smaller $S_{F R}$ implies higher $Z_{\text {end }}$.

In our assumption of beginning strangulation, stars continue to form and consume the available gas in the disk either with a constant SFR (blue tracks in Fig. 9) or with a slowly decreasing SFR on a timescale $\tau$ of 3 Gyr (black tracks in Fig. 9). We find that a model galaxy with an exponentially faster declining SFR with a $\tau=1$ Gyr becomes passive by $z \sim 0.06$ with a SSFR about 30 times lower than at $z \sim 0.4$, therefore disappearing from panel a of Fig. 9. Recent results of Cantale et al. (2016) strengthen the plausibility of our toy models with ongoing star formation for several Gyr since they found that galaxies are able to continue forming stars for up to $5 \mathrm{Gyr}$ after being accreted into clusters. Additionally, Jaffé et al. (2015) studied HI gas masses in cluster galaxies at $z \sim 0.2$ and found a plausible scenario in which galaxies affected by ram-pressure stripping as they first fall into the cluster are able to continue to form stars in the remaining gas disk.

In the strangulation scenario, our model galaxy reaches the same values of $\log M_{\text {end }}$ and SFR $_{\text {end }}$ as above, but the increase in metallicity is higher than in the non-strangulation case, i.e., $Z_{\text {end }}$ is higher. After stopping the inflow of gas, the evolution of the galaxy can be approximated with a closed-box model. This first-order assumption neglects effects of gas outflows that continue after the supply of cold gas from the surroundings comes to a halt. However, studies have shown (e.g., Peng et al. 2015) that in the case of truncated gas inflows, metallicities are independent of outflows, because the element mix of the retained gas stays constant. Thus, a closed-box model is still a good approximation. Maier et al. (2006) constructed a large grid of Pégase 2 models to explore which region of the parameter space could reproduce the constraints imposed by the local metallicityluminosity relation and by the metallicities and luminosities of galaxies at higher redshifts. They explored models in which the gas inflow on the model galaxy stops after about $1 \mathrm{Gyr}$. The galaxy subsequently forms stars in a "closed-box"-like scenario. The tracks of these closed-box models show that, after gas inflow stops, the model galaxies increase their metallicities by $\sim 0.2 \mathrm{dex}$ in $t_{\text {enrich }} \sim 1$ Gyr and by more than a factor of two, namely by $\sim 0.35 \mathrm{dex}$, in $t_{\text {enrich }} \sim 3 \mathrm{Gyr}$.

In both strangulation scenarios, we therefore assume a metallicity increase of more than a factor of two in $t_{\text {enrich }} \sim 3 \mathrm{Gyr}$, as justified by the Pégase 2 closed-box models, which leads to final metallicity values of $Z_{\text {end }}=8.9$ (black and blue tracks in Fig. 9). These $Z_{\text {end }}$ values of the model starvation paths lie in the region of the mass-metallicity diagram populated by higher metallicity SDSS objects (the metallicity of the red symbols in panel $b$ in Fig. 9 are in agreement with the local SDSS MZR). This indicates that starvation is a plausible scenario for the evolution of the metallicities of $z \sim 0.4$ infalling galaxies to higher metallicities after $3 \mathrm{Gyr}$, consistent with the upper, higher metallicity part of the $z \sim 0.06 \mathrm{MZR}$. Additionally, the $Z_{\text {end }}$ values of the model starvation paths are also in agreement with the $\mathrm{O} / \mathrm{Hs}$ of several accreted cluster galaxies at $z \sim 0.4$ (red filled circles in Fig. 9). Therefore, $z \sim 0.4$ accreted cluster members with higher metallicities are likely to have been infalling into the cluster at earlier times $(z \sim 0.8)$ and to have suffered strangulation in the time span of about 3 Gyr between $z \sim 0.8$ and $z \sim 0.4$.

\section{Summary}

This study of environmental effects on the $Z(M, \mathrm{SFR})$ relation is based on VIMOS spectroscopy of cluster galaxies in the MAC0416 cluster, supplemented by a zCOSMOS field galaxy sample at similar redshifts $z \sim 0.4$. The main results can be summarized as follows:

1. The star-forming cluster galaxies at $z \sim 0.4$ are not dominated by AGNs, and they follow the star-forming abundance sequence in the BPT diagram (Fig. 2).

2. Cluster and field galaxies at $z \sim 0.4$ show, on average, similar MZR and mass-sSFR relations for stellar masses $9.2<\log \left(M / M_{\odot}\right)<10.2$ (Figs. 3 and 5), in accordance with the findings of numerical studies and local observations. On the other hand, we find that bulge-dominated (smooth) and compact cluster galaxies have lower sSFRs and higher $\mathrm{O} / \mathrm{Hs}$ than their disk-dominated and peculiar counterparts (Figs. 4 and 6) in agreement with an FMR scenario combined with the effect of dominant bulges lowering SFRs.

3. We find several indications for a strangulation scenario for galaxies with $\log \left(M / M_{\odot}\right)<10.2$ infalling into a cluster:

i) First, based on the location of galaxies in the projected phase-space diagram, a higher fraction of accreted galaxies $(63 \%)$ are metal-rich compared to the fraction of $28 \%$ of metal-rich infalling galaxies (Fig. 7). 
ii) Second, while the measured $\mathrm{O} / \mathrm{Hs}$ of field and infalling cluster galaxies are in quite good agreement with the FMR, accreted galaxies have a distribution shifted to higher metallicities than predicted by the Li13 models (Fig. 8). This trend is intensified for low-mass $\left(\log \left(M / M_{\odot}\right)<9.2\right)$ accreted cluster galaxies, which have 2-3 times higher metallicities than predicted by the models with inflowing pristine gas, indicating that a strangulation scenario in which gas inflow has stopped enhancing their metallicities is a plausible mechanism.

iii) Third, the paths of model galaxies in the MZR and in the $\triangle S S F R-\triangle \mathrm{O} / \mathrm{H}$ diagrams show that strangulation is needed, in addition to a constant or exponentially declining SFR, to explain the higher metallicities of accreted cluster galaxies (Fig. 9).

The main conclusion is that a strangulation scenario is plausible to explain the metallicities of infalling and accreted $\log \left(M / M_{\odot}\right)<10.2$ cluster galaxies in MAC0416. While the $Z(M, \mathrm{SFR})$ measurements of most field and infalling cluster galaxies are in agreement with the FMR assuming a primordial gas inflow, we find higher values than from the FMR expected metallicities in accreted cluster galaxies, especially at lower masses, indicating that the inflow of pristine gas is being stopped in these galaxies as a result of cluster environmental effects.

Acknowledgements. We would like to thank the anonymous referee for providing constructive comments and help in improving the manuscript. This publication is supported by the Austrian Science Fund (FWF). I.B., A.M., P.R., M.N., and B.S. acknowledge support from PRIN-INAF 2014 1.05.01.94.02 (PI M. Nonino).

\section{References}

Abramson, L. E., Kelson, D .D., Dressler, A., et al. 2014, ApJ, 785, 36 Arnouts, S., \& Ilbert, O. 2011, Astrophysics Source Code Library [record ascl:1108.009]

Baldwin, J. A., Phillips, M. M., \& Terlevich, R. 1981, PASP, 93, 5

Balestra, I., Mercurio, A., Sartoris, B., et al. 2016, ApJS, submitted [arXiv: 1511.02522] (B16)

Bamford, S. P., Häußler, B, Rojas, A., \& Borch, A. 2011, ASP Conf. Ser., 442, 479

Brinchmann, J., Charlot, S., White, S. D. M., et al. 2004, MNRAS, 351, 1151

Bruzual, G., \& Charlot, S. 2003, MNRAS, 344, 1000

Cresci, G., Mannucci, F., Sommariva, V., et al. 2012, MNRAS, 421, 262

Calura, F., Pipino, A., Chiappini, C., Matteucci, F., \& Maiolino, R. 2009, A\&A, 504,373

Cantale, N., Jablonka, P., Courbin, F., et al. 2016, A\&A, 589, A82

Chabrier G. 2003, PASP, 115, 763

Cooper, M. C., Tremonti, C. A., Newman, J. A., \& Zabludoff, A. I. 2008, MNRAS, 390, 245

Cox, T. J., Jonsson, P., Somerville, R. S., Primack, J. R., \& Dekel, A. 2008, MNRAS, 384, 386

Davé, R., Finlator, K., \& Oppenheimer, B. D. 2011, MNRAS, 416, 1354

Davé, R., Finlator, K., \& Oppenheimer, B. D. 2012, MNRAS, 421, 98

Dayal, P., Ferrara, A., \& Dunlop, J. S. 2013, MNRAS, 430, 289

Dressler, A. 1980, ApJ, 236, 351

Ebeling, H., Edge, A. C., \& Henry, J. P., 2001, ApJ, 553, 668

Elbaz, D., Daddi, E., Le Borgne, D., et al. 2007, A\&A, 468, 33

Ellison, S. L., Patton, D. R., Simard, L., \& McConnachie, A. W. 2008, ApJ, 672, L107
Ellison, S. L., Simard, L., Cowan, N. B., et al. 2009, MNRAS, 396, 1257 Häußler, B., Bamford, S. P., Vika, M., et al. 2013, MNRAS, 430, 330 Haines, C. P., Pereira, M. J., Smith, G. P., et al. 2015, ApJ, 806, 101 Hubble E. P. 1926, ApJ, 64, 321

Ilbert O., Capak, P., Salvato, M., et al. 2009, ApJ, 690, 1236

Jaffé, Y. L., Smith, R., Candlish, G., et al. 2015, MNRAS, 448, 1715

Kauffmann, G., Heckman, T. M., Tremonti. C., et al. 2003a, MNRAS, 346, 1055

Kauffmann, G., Heckman, T. M., White, S. D. M., et al. 2003b, MNRAS, 341, 33

Kennicutt, R. C., Jr. 1998, ARA\&A, 36, 189

Kewley, L. J., \& Ellison, S. L. 2008, ApJ, 681, 1183

Kewley, L. J., \& Dopita, M. A. 2002, ApJSS, 142, 35

Kewley, L. J., Jansen, R. A., \& Geller, M. J. 2005, PASP, 117, 227

Kewley, L. J. Heisler, C. A., Dopita, M. A., Lumsden, S. 2001, ApJS, 132, 37

Kewley, L. J., Dopita, M. A., Leitherer, C., et al. 2013, ApJ, 774, 100

Kobulnicky, H. A., Kennicutt, R. C., Jr., \& Pizagno, J. L. 1999, ApJ, 514, 544,

Kormendy, J., Fisher, D. B., Cornell, M. E., \& Bender, R. 2009, ApJS, 182, 216

Larson, R. B., Tinsley, B. M., \& Caldwell, C. N. 1980, ApJ, 237, 692

Lara-López, M. A., Cepa, J., Bongiovanni, A., et al. 2010, A\&A, 521, A53

Lilly, S. J, Carollo, C. M., \& Stockton, A. 2003, ApJ, 597, 730

Lilly, S. J., Le Fèvre, O., Renzini, A., et al. 2007, ApJS, 172, 70

Lilly, S. J., Le Brun, V., Maier, C., et al. 2009, ApJS, 184, 218

Lilly, S. J., Carollo, C. M., Pipino, A., Renzini, A., Peng, Y. 2013, ApJ, 772, 119

Maier, C., Meisenheimer, K., \& Hippelein, H. 2004, A\&A, 418, 475

Maier, C., Lilly, S. J., Carollo, C. M., et al. 2005, ApJ, 634, 849

Maier, C., Lilly, S. J., Carollo, C. M., et al. 2006, ApJ, 639, 858

Maier, C., Lilly, S. J., Zamorani, G., et al. 2009, ApJ, 694, 1099

Maier, C., Lilly, S. J., Ziegler, B. L., et al. 2014, ApJ, 792, 3

Maier, C., Ziegler, B. L., Lilly, S. J., et al. 2015, A\&A, 577, A14

Mancone, C. L., \& Gonzalez, A. H. 2012, PASP, 124, 606

Mannucci, F., Cresci, G., Maiolino, R., Marconi, A., \& Gnerucci, A. 2010, MNRAS, 408, 2115

Mihos, J. C., \& Hernquist, L. 1994, ApJ, 425, L13

Mihos, J. C., \& Hernquist, L. 1996, ApJ, 464, 641

Mouhcine, M., Baldry, I. K., \& Bamford, S. P. 2007, MNRAS, 382, 801

Moustakas, J., Coil, A. L., Aird, J, et al. 2013, ApJ, 767, 50

Noeske, K. G., Weiner, B. J., Faber, S. M., et al. 2007, ApJ, 660, 43

Nantais, J. B., Flores, H., Demarco, R., et al. 2013, A\&A, 555, A5

Neichel, B., Hammer, F., Puech, M., et al. 2008, A\&A, 484, 159

Ogrean, G. A., van Weeren, R. J., Jones, C., et al. 2015, ApJ, 812, 153

Pasquali, A., Gallazzi, A., \& van den Bosch, F. C. 2012, MNRAS, 425, 273

Peng, Y.-J., \& Maiolino, R. 2014, MNRAS, 438, 262

Peng, Y.-J., Lilly, S. J., Kovač, K., et al. 2010a, ApJ, 721, 193

Peng, C. Y., Ho, L. C., Impey, C. D., \& Rix, H.-W. 2010b, AJ, 139, 2097

Peng, Y.-J., Lilly, S. J., Renzini, A., \& Carollo, M. 2012, ApJ, 757, 4

Peng, Y., Maiolino, R., \& Cochrane, R. 2015, Nature, 521, 192

Pérez-Montero, E., Contini, T., Lamareille, F., et al. 2013, A\&A, 549, A25

Pérez, E., Cid Fernandes, R., González Delgado, R. M., et al. 2013, ApJ, 764, L1

Pettini, M., \& Pagel, B. E. J. 2004, MNRAS, 348, 59

Pipino, A., Lilly, S. J., \& Carollo, C. M. 2014, MNRAS, 441, 1444

Postman, M., Coe, M., Benitez, N., et al. 2012, ApJS, 199, 25

Pozzetti, L., Bolzonella, M., Lamareille, F., et al. 2007, A\&A, 474, 443

Renzini, A., \& Peng, Y. 2015, ApJ, 801, 29

Rosati, P., Balestra, I., Grillo, C., et al. 2014, The Messenger, 158, 48

Saintonge, A., Tacconi, L. J., Fabello, S., et al. 2012, ApJ, 758, 73

Salim, S., Rich, R. M., Charlot, S., et al. 2007, ApJS, 173, 267

Salim, S., Lee, J. C., Ly, C., et al. 2014, ApJ, 797, 126

Salpeter, E. E. 1955, ApJ, 121, 161

Scodeggio, M., Franzetti, P., Garilli, B., et al. 2005, PASP, 117, 1284

Simard, L., Mendel, J. T., Patton, D. R., et al. 2011, ApJS, 196, 11

Tremonti, C. A., Heckman, T. M., Kauffmann, G., et al. 2004, ApJ, 613, 898

Umetsu, K., Medezinski, E., Nonino, M., et al. 2014, ApJ, 795, 163

Vika, M., Bamford, S. P., Häußler, B., \& Rojas, A. L. 2014, MNRAS, 444, 3603

Vila-Costas M. B., \& Edmunds M. G. 1992, MNRAS, 259, 121

Whitaker, K., Franx, M., Bezanson, R., et al. 2015, ApJ, 811, 12

Zaritsky, D. 1993, PASP, 105, 1006

Zaritsky D., Kennicutt R. C., Jr, Huchra J. P. 1994, ApJ, 420, 87

Zabludoff, A. I., \& Mulchaey, J. S. 1998, ApJ, 496, 39 
A\&A 590, A108 (2016)

\section{Appendix A: Additional table}

Table A.1. Observed and derived quantities for the MAC0416 $z \sim 0.4$ cluster galaxies.

\begin{tabular}{|c|c|c|c|c|c|c|c|}
\hline Id & $z$ & $\mathrm{H} \beta$ & [O III] $] \lambda 5007$ & $\mathrm{H} \alpha$ & {$[\mathrm{N} \mathrm{III}] \lambda 6584$} & $\mathrm{O} / \mathrm{H}$ & $\log \left(M / M_{\odot}\right)$ \\
\hline 20904 & 0.4007 & $9.75 \pm 0.97$ & $6.48 \pm 0.68$ & $35.82 \pm 1.71$ & $10.23 \pm 1.71$ & $8.85 \pm 0.03$ & $9.92_{+0.04}^{-0.19}$ \\
\hline 24665 & 0.3907 & $7.97 \pm 0.63$ & $13.90 \pm 0.48$ & $25.17 \pm 1.20$ & $1.68 \pm 0.24$ & $8.52 \pm 0.02$ & $9.55^{+0.13} \begin{array}{r}-0.13 \\
+0.04\end{array}$ \\
\hline 25506 & 0.3844 & $5.04 \pm 0.63$ & $15.15 \pm 0.64$ & $17.57 \pm 0.97$ & $1.93 \pm 0.32$ & $8.51 \pm 0.03$ & $9.18_{+0.03}^{-0.03}$ \\
\hline 25980 & 0.3858 & $4.69 \pm 0.84$ & $2.05 \pm 0.59$ & $14.64 \pm 1.17$ & $4.69 \pm 0.88$ & $8.93 \pm 0.05$ & $9.88_{+0.03}^{-0.04}$ \\
\hline 27930 & 0.4048 & $6.82 \pm 0.53$ & $12.01 \pm 0.42$ & $18.96 \pm 0.84$ & $3.37 \pm 0.84$ & $8.65 \pm 0.04$ & $9.92_{+0.05}^{-0.02}$ \\
\hline 28862 & 0.3992 & $6.85 \pm 0.53$ & $16.59 \pm 0.46$ & $18.90 \pm 0.92$ & $1.38 \pm 0.23$ & $8.48 \pm 0.03$ & $9.14_{+0.04}^{-0.04}$ \\
\hline 31765 & 0.3931 & $2.50 \pm 0.22$ & $6.94 \pm 0.35$ & $8.33 \pm 0.69$ & $0.52 \pm 0.17$ & $8.44 \pm 0.05$ & $8.98_{+0.08}^{-0.04}$ \\
\hline 32731 & 0.4034 & $4.93 \pm 0.50$ & $21.03 \pm 0.44$ & $12.71 \pm 0.66$ & $0.66 \pm 0.22$ & $8.36 \pm 0.05$ & $8.78_{+0.11}^{-0.02}$ \\
\hline 33061 & 0.3839 & $1.63 \pm 0.25$ & $6.32 \pm 0.33$ & $4.58 \pm 0.44$ & $0.22 \pm 0.22$ & $8.36 \pm 0.14$ & $8.79_{+0.17}^{-0.11}$ \\
\hline 33592 & 0.3846 & $6.70 \pm 1.60$ & $2.73 \pm 0.41$ & $29.79 \pm 1.37$ & $10.93 \pm 1.37$ & $8.96 \pm 0.04$ & $10.45_{+0.01}^{-0.17}$ \\
\hline 43168 & 0.3899 & $2.07 \pm 0.26$ & $4.27 \pm 0.21$ & $5.33 \pm 0.64$ & $0.43 \pm 0.21$ & $8.52 \pm 0.07$ & $8.93_{+0.08}^{-0.09}$ \\
\hline 47541 & 0.3903 & $6.81 \pm 0.63$ & $13.42 \pm 0.79$ & $23.67 \pm 1.32$ & $2.10 \pm 0.26$ & $8.54 \pm 0.02$ & $9.65^{-0.21}+0.04$ \\
\hline 48262 & 0.4040 & $1.98 \pm 0.40$ & $0.66 \pm 0.11$ & $7.25 \pm 0.44$ & $1.76 \pm 0.22$ & $8.93 \pm 0.04$ & $9.51_{+0.03}^{-0.24}$ \\
\hline 54317 & 0.3928 & $11.92 \pm 1.20$ & $25.00 \pm 0.63$ & $33.13 \pm 1.25$ & $3.44 \pm 0.63$ & $8.55 \pm 0.03$ & $9.48_{+0.05}^{-0.03}$ \\
\hline 55515 & 0.3934 & $7.98 \pm 1.49$ & $2.22 \pm 0.28$ & $34.08 \pm 2.77$ & $10.25 \pm 1.11$ & $8.98 \pm 0.04$ & $10.15_{+0.21}^{-0.02}$ \\
\hline 60453 & 0.3875 & $16.85 \pm 0.81$ & $15.26 \pm 0.66$ & $51.70 \pm 1.64$ & $15.59 \pm 1.64$ & $8.82 \pm 0.02$ & $10.17_{+0.05}^{-0.02}$ \\
\hline 61634 & 0.3843 & $11.06 \pm 0.65$ & $21.31 \pm 0.81$ & $35.30 \pm 0.81$ & $4.07 \pm 0.81$ & $8.58 \pm 0.03$ & $9.73_{+0.04}^{-0.03}$ \\
\hline 62697 & 0.3814 & $5.76 \pm 2.12$ & $1.77 \pm 0.55$ & $26.82 \pm 1.33$ & $9.98 \pm 1.11$ & $9.00 \pm 0.07$ & $10.32^{+0.04}-0.12$ \\
\hline 64825 & 0.4026 & $7.52 \pm 0.92$ & $14.06 \pm 0.66$ & $20.76 \pm 1.31$ & $2.50 \pm 0.39$ & $8.59 \pm 0.03$ & $9.56_{+0.04}^{-0.12}$ \\
\hline 65028 & 0.3844 & $5.90 \pm 0.47$ & $11.22 \pm 0.64$ & $18.21 \pm 0.64$ & $1.91 \pm 0.32$ & $8.57 \pm 0.03$ & $\begin{array}{r}9.27^{-0.23}+0.00 \\
+0.04\end{array}$ \\
\hline 68953 & 0.4092 & $2.54 \pm 0.38$ & $1.35 \pm 0.25$ & $9.82 \pm 0.85$ & $2.71 \pm 0.51$ & $8.88 \pm 0.04$ & $\begin{array}{r}9.79_{+0.13}^{-0.13}+04 \\
+0.04\end{array}$ \\
\hline 69388 & 0.3836 & $3.55 \pm 0.38$ & $6.99 \pm 0.33$ & $12.03 \pm 0.98$ & $1.46 \pm 0.49$ & $8.58 \pm 0.05$ & $9.45_{+0.01}^{-0.33}$ \\
\hline 69442 & 0.3951 & $26.39 \pm 1.12$ & $23.83 \pm 0.78$ & $75.12 \pm 2.59$ & $14.76 \pm 1.30$ & $8.76 \pm 0.02$ & $9.84_{+0.03}^{-0.04}$ \\
\hline 70675 & 0.4108 & $3.84 \pm 0.41$ & $11.52 \pm 0.35$ & $12.74 \pm 0.70$ & $0.87 \pm 0.17$ & $8.44 \pm 0.03$ & $9.22_{+0.05}^{-0.02}$ \\
\hline 72228 & 0.3894 & $2.12 \pm 0.21$ & $12.15 \pm 0.37$ & $6.99 \pm 0.28$ & $0.37 \pm 0.09$ & $8.32 \pm 0.04$ & $8.48_{+0.08}^{-0.05}$ \\
\hline 73404 & 0.3954 & $5.97 \pm 0.50$ & $4.41 \pm 0.37$ & $19.29 \pm 0.92$ & $4.41 \pm 0.55$ & $8.81 \pm 0.02$ & $9.51_{+0.06}^{-0.03}$ \\
\hline 74065 & 0.4002 & $5.91 \pm 0.54$ & $14.94 \pm 0.47$ & $16.11 \pm 0.70$ & $1.40 \pm 0.23$ & $8.50 \pm 0.03$ & $9.31_{+0.11}^{-0.16}$ \\
\hline 76447 & 0.4084 & $14.16 \pm 1.31$ & $24.84 \pm 0.87$ & $44.22 \pm 1.31$ & $6.32 \pm 1.31$ & $8.62 \pm 0.03$ & $10.04_{+0.02}^{-0.11}$ \\
\hline 78137 & 0.3932 & $14.01 \pm 4.88$ & $9.57 \pm 0.93$ & $63.05 \pm 4.67$ & $25.69 \pm 2.34$ & $8.90 \pm 0.05$ & $10.84_{+0.02}^{-0.02}$ \\
\hline 80425 & 0.4090 & $5.87 \pm 1.11$ & $7.22 \pm 0.60$ & $14.45 \pm 0.90$ & $1.81 \pm 0.30$ & $8.65 \pm 0.04$ & $9.64_{+0.03}^{-0.05}$ \\
\hline 80427 & 0.4088 & $10.58 \pm 1.00$ & $8.40 \pm 0.51$ & $40.89 \pm 1.01$ & $11.33 \pm 0.81$ & $8.82 \pm 0.02$ & $10.10_{+0.05}^{-0.04}$ \\
\hline 82461 & 0.3865 & $4.81 \pm 0.43$ & $11.19 \pm 0.60$ & $14.77 \pm 1.19$ & $1.07 \pm 0.24$ & $8.49 \pm 0.04$ & $8.94_{+0.09}^{-0.03}$ \\
\hline 84626 & 0.3967 & $3.38 \pm 0.40$ & $10.94 \pm 0.50$ & $12.26 \pm 0.83$ & $1.08 \pm 0.17$ & $8.47 \pm 0.03$ & $8.90_{+0.03}^{-0.08}$ \\
\hline 85922 & 0.3935 & $5.60 \pm 0.61$ & $18.92 \pm 0.66$ & $17.60 \pm 0.88$ & $1.10 \pm 0.22$ & $8.42 \pm 0.03$ & $8.71_{+0.03}^{-0.09}$ \\
\hline 87340 & 0.3939 & $12.37 \pm 2.96$ & $2.52 \pm 0.76$ & $49.73 \pm 1.77$ & $22.72 \pm 1.26$ & $9.08 \pm 0.05$ & $10.83_{+0.04}^{-0.03}$ \\
\hline 92461 & 0.3917 & $4.68 \pm 0.71$ & $3.12 \pm 0.32$ & $13.56 \pm 0.65$ & $3.87 \pm 0.65$ & $8.85 \pm 0.04$ & $10.11_{+0.05}^{-0.02}$ \\
\hline 92623 & 0.3922 & $3.56 \pm 0.76$ & $6.24 \pm 0.56$ & $12.47 \pm 0.89$ & $3.34 \pm 0.67$ & $8.71 \pm 0.04$ & $9.46_{+0.02}^{-0.06}$ \\
\hline 92946 & 0.3998 & $6.54 \pm 1.49$ & $6.61 \pm 0.43$ & $20.99 \pm 0.86$ & $8.63 \pm 1.44$ & $8.84 \pm 0.04$ & $9.90_{+0.06}^{-0.01}$ \\
\hline 93590 & 0.3940 & $4.11 \pm 0.43$ & $1.42 \pm 0.32$ & $14.18 \pm 0.63$ & $3.78 \pm 0.63$ & $8.93 \pm 0.04$ & $10.08_{+0.02}^{-0.17}$ \\
\hline 95694 & 0.4071 & $8.17 \pm 0.48$ & $14.60 \pm 0.40$ & $22.00 \pm 0.80$ & $1.60 \pm 0.40$ & $8.53 \pm 0.04$ & $\begin{array}{l}9.32_{+0.43}^{-0.00} \\
\end{array}$ \\
\hline
\end{tabular}

Notes. EL fluxes are given in $10^{-17} \mathrm{erg} \mathrm{s}^{-1} \mathrm{~cm}^{-2}$. The $\mathrm{H} \beta$ flux was corrected for stellar absorption as described in Sect. 3.1, and all fluxes in the table were corrected for slit losses as described in Sect. 3.6. The stellar mass uncertainties were computed as described in Sect. 3.4. 
Table A.1. continued.

\begin{tabular}{|c|c|c|c|c|c|c|c|}
\hline Id & $z$ & $\mathrm{H} \beta$ & [O III] $\lambda 5007$ & $\mathrm{H} \alpha$ & {$[\mathrm{N} \mathrm{II}] \lambda 6584$} & $\mathrm{O} / \mathrm{H}$ & $\log \left(M / M_{\odot}\right)$ \\
\hline 105374 & .5990 & $8.54 \pm 0.82$ & $.36 \pm 0.34$ & $59.4 J \pm 1.01$ & $11.13 \pm 1.01$ & $.97 \pm 0.03$ & $10.31_{+0.00}^{-0.27}$ \\
\hline 106119 & 0.4058 & $8.09 \pm 0.83$ & $23.92 \pm 1.01$ & $25.27 \pm 1.01$ & $0.67 \pm 0.34$ & $8.32 \pm 0.07$ & $9.28_{+0.04}^{-0.03}$ \\
\hline 107694 & 0.3975 & $1.54 \pm 0.24$ & $4.73 \pm 0.19$ & $5.98 \pm 0.58$ & $0.39 \pm 0.19$ & $8.43 \pm 0.07$ & $8.75_{+0.12}^{-0.04}$ \\
\hline 108060 & 0.3975 & $6.44 \pm 1.71$ & $5.23 \pm 0.58$ & $25.19 \pm 1.94$ & $8.52 \pm 1.16$ & $8.85 \pm 0.05$ & $9.90_{+0.03}^{-0.05}$ \\
\hline 110267 & 0.3964 & $3.68 \pm 1.43$ & $1.84 \pm 0.18$ & $16.92 \pm 1.47$ & $4.78 \pm 1.10$ & $8.89 \pm 0.07$ & $10.08_{+0.03}^{-0.08}$ \\
\hline 110762 & 0.3851 & $2.50 \pm 0.47$ & $6.08 \pm 0.54$ & $7.51 \pm 0.72$ & $0.36 \pm 0.36$ & $8.42 \pm 0.14$ & $8.86_{+0.09}^{-0.04}$ \\
\hline 113035 & 0.4022 & $4.76 \pm 2.16$ & $0.95 \pm 0.95$ & $17.87 \pm 1.08$ & $4.60 \pm 0.81$ & $9.01 \pm 0.15$ & $10.48_{+0.04}^{-0.03}$ \\
\hline 115312 & 0.3892 & $6.09 \pm 0.93$ & $3.42 \pm 0.43$ & $20.95 \pm 1.50$ & $4.70 \pm 1.07$ & $8.84 \pm 0.04$ & 9.96 \\
\hline 116392 & 0.3959 & & & & & $8.73 \pm 0.03$ & $9.42_{+0.05}^{-0.13}$ \\
\hline 118060 & 0.3836 & $3.62 \pm 0.56$ & $1.66 \pm 0.25$ & $14.79 \pm 1.02$ & $3.95 \pm 0.64$ & $8.90 \pm 0.04$ & $\begin{array}{r}9.71_{+0.19}^{-0.03} \\
+0.19\end{array}$ \\
\hline 119315 & 0.4007 & $14.41 \pm 1.26$ & $41.30 \pm 1.75$ & $46.90 \pm 3.50$ & $4.20 \pm 1.40$ & $8.49 \pm 0.05$ & $9.26_{+0.05}^{-0.02}$ \\
\hline 119393 & 0.4001 & $2.26 \pm 0.42$ & $1.10 \pm 0.16$ & $9.28 \pm 0.63$ & $3.30 \pm 0.31$ & $8.93 \pm 0.04$ & $9.34_{+0.02}^{-0.04}$ \\
\hline 120959 & 0.3822 & $10.39 \pm 0.60$ & $30.12 \pm 0.53$ & $38.52 \pm 0.88$ & $3.33 \pm 0.35$ & $8.48 \pm 0.02$ & 9.71 \\
\hline 121798 & 0.3962 & $26.35 \pm 1.91$ & $31.40 \pm 0.86$ & $102.76 \pm 2.85$ & $20.27 \pm 2.00$ & $8.72 \pm 0.02$ & 10.13 \\
\hline 122137 & 0.3969 & $5.20 \pm 0.59$ & $14.43 \pm 0.72$ & $14.43 \pm 0.96$ & $1.32 \pm 0.36$ & $8.50 \pm 0.04$ & 8.96 \\
\hline 122217 & 0.4013 & $5.37 \pm 0.78$ & $8.32 \pm 0.54$ & $15.56 \pm 1.07$ & $1.34 \pm 0.27$ & $8.57 \pm 0.04$ & $9.38_{+0.08}^{-0.03}$ \\
\hline 122730 & 0.3909 & $3.99 \pm 0.90$ & $2.28 \pm 0.25$ & $11.31 \pm 0.84$ & $2.53 \pm 0.84$ & $8.84 \pm 0.06$ & $9.77_{+0.04}^{-0.04}$ \\
\hline 123859 & 0.3956 & $1.62 \pm 0.60$ & $3.24 \pm 0.27$ & $9.18 \pm 0.54$ & $1.89 \pm 0.27$ & $8.65 \pm 0.06$ & $9.55_{+0.03}^{-0.05}$ \\
\hline 126188 & 0.3943 & $2.72 \pm 0.36$ & $13.11 \pm 0.34$ & $7.65 \pm 0.25$ & & $8.32 \pm$ & 8.52 \\
\hline 127932 & 3949 & $5.08 \pm 0.62$ & $5.08 \pm 0.30$ & $15.23 \pm 0.81$ & $3.65 \pm 0.81$ & $8.77 \pm 0.04$ & 9.69 \\
\hline 128401 & 0.3904 & $1.99 \pm 0.74$ & $1.16 \pm 0.17$ & $6.80 \pm 0.66$ & $2.16 \pm 0.33$ & $8.89 \pm 0.06$ & $9.87_{+0.06}^{-0.05}$ \\
\hline 128987 & 0.3957 & $4.48 \pm 0.59$ & $4.08 \pm 0.31$ & $16.47 \pm 0.78$ & $5.18 \pm 0.63$ & $8.82 \pm 0.03$ & $9.90_{+0.06}^{-0.02}$ \\
\hline 130603 & 0.3997 & $3.18 \pm 0.32$ & $8.55 \pm 0.40$ & $11.73 \pm 0.60$ & $1.19 \pm 0.20$ & $8.51 \pm 0.03$ & $9.28_{+0.04}^{-0.09}$ \\
\hline 131120 & 0.3954 & $1.33 \pm 0.20$ & $2.31 \pm 0.18$ & $4.70 \pm 0.44$ & $0.53 \pm 0.18$ & $8.59 \pm 0.05$ & $9.18_{+0.12}^{-0.15}$ \\
\hline 131278 & & & & & & & \\
\hline 132372 & 0.4022 & $1.47 \pm 0.41$ & $2.14 \pm 0.15$ & $5.05 \pm 0.31$ & $0.69 \pm 0.15$ & $8.64 \pm 0.05$ & $\begin{array}{l}9.72^{-0.15}+0.04 \\
+0.15\end{array}$ \\
\hline 133372 & 0.3834 & $3.38 \pm 0.23$ & $5.89 \pm 0.29$ & $12.53 \pm 0.59$ & $1.03 \pm 0.29$ & $8.55 \pm 0.04$ & $9.40_{+0.06}^{-0.16}$ \\
\hline 136237 & 0.3950 & $2.50 \pm 0.93$ & $2.71 \pm 0.21$ & $11.69 \pm 1.25$ & $2.50 \pm 0.63$ & $8.74 \pm 0.07$ & $10.06_{+0.02}^{-0.27}$ \\
\hline 140223 & 0.3967 & $3.99 \pm 0.37$ & $2.99 \pm 0.33$ & $15.94 \pm 0.66$ & $4.65 \pm 0.66$ & $8.84 \pm 0.03$ & $9.80_{+0.17}^{-0.03}$ \\
\hline 141465 & 0.3944 & $2.72 \pm 0.31$ & $4.29 \pm 0.19$ & $8.96 \pm 0.47$ & $1.31 \pm 0.37$ & $8.64 \pm 0.04$ & $9.05_{+0.15}^{-0.11}$ \\
\hline 141596 & 0.3978 & $3.74 \pm 0.43$ & $3.15 \pm 0.26$ & $15.99 \pm 0.85$ & $4.42 \pm 0.68$ & $8.82 \pm 0.03$ & $9.68_{+0.00}^{-0.41}$ \\
\hline 144037 & 0.4016 & $8.01 \pm 0.68$ & $13.21 \pm 0.88$ & $28.61 \pm 2.20$ & $5.94 \pm 0.66$ & $8.68 \pm 0.02$ & $\begin{array}{r}9.55^{-0.05}+0.02 \\
+02\end{array}$ \\
\hline 144528 & 0.4021 & $6.12 \pm 0.51$ & $14.62 \pm 0.28$ & $21.09 \pm 1.41$ & $2.67 \pm 0.28$ & $8.56 \pm 0.02$ & $9.38_{+0.05}^{-0.04}$ \\
\hline 144606 & 0.3993 & $4.05 \pm 0.45$ & $1.21 \pm 0.26$ & $18.18 \pm 0.87$ & $6.93 \pm 0.69$ & $9.00 \pm 0.04$ & 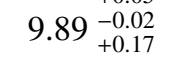 \\
\hline 148186 & 0.3981 & $2.35 \pm 0.24$ & $7.15 \pm 0.39$ & $6.95 \pm 0.39$ & $0.58 \pm 0.19$ & $8.47 \pm 0.05$ & $\begin{array}{l}8.95_{+0.14}^{-0.05} \\
+0.00\end{array}$ \\
\hline 148883 & 0.3970 & $5.40 \pm 0.47$ & $9.16 \pm 0.35$ & $22.46 \pm 0.69$ & $3.46 \pm 0.52$ & $8.64 \pm 0.03$ & $\begin{array}{r}9.90_{+0.00}^{-0.36} \\
\end{array}$ \\
\hline
\end{tabular}

